\title{
Many-particle Hamiltonian for open systems with full Coulomb interaction: Application to classical and quantum time-dependent simulations of nanoscale electron devices
}

\author{
G. Albareda, J. Suñé, and X. Oriols* \\ Departament d'Enginyeria Electrònica, Universitat Autònoma de Barcelona, 08193 Bellaterra, Spain
}

(Received 12 July 2008; published 17 February 2009)

\begin{abstract}
A many-particle Hamiltonian for a set of particles with Coulomb interaction inside an open system is described without any perturbative or mean-field approximation. The boundary conditions of the Hamiltonian on the borders of the open system [in the real three-dimensional (3D) space representation] are discussed in detail to include the Coulomb interaction between particles inside and outside of the open system. The manyparticle Hamiltonian provides the same electrostatic description obtained from the image-charge method, but it has the fundamental advantage that it can be directly implemented into realistic (classical or quantum) electron device simulators via a 3D Poisson solver. Classically, the solution of this many-particle Hamiltonian is obtained via a coupled system of Newton-type equations with a different electric field for each particle. The quantum-mechanical solution of this many-particle Hamiltonian is achieved using the quantum (Bohm) trajectory algorithm [X. Oriols, Phys. Rev. Lett. 98, 066803 (2007)]. The computational viability of the manyparticle algorithms to build powerful nanoscale device simulators is explicitly demonstrated for a (classical) double-gate field-effect transistor and a (quantum) resonant tunneling diode. The numerical results are compared with those computed from time-dependent mean-field algorithms showing important quantitative differences.
\end{abstract}

DOI: 10.1103/PhysRevB.79.075315

PACS number(s): 73.23.-b, 71.10.-w, 02.70.-c, 41.20.Cv

\section{INTRODUCTION}

The exact computation of a system of interacting electrons is extremely complicated ${ }^{1,2}$ because the motion of one electron depends on the positions of all others (i.e., electrons are correlated $\left.{ }^{3}\right)$. Thus, the prediction of the collective behavior of many electrons is still a very active field of research in nanoelectronics, quantum chemistry, nanobiology, quantum computing, materials science, etc. Several theoretical approximations have been proposed to improve the treatment of electron-electron correlations.

In quantum systems in equilibrium, the time-independent mean-field approximation appears as a successful solution to treat a set of interacting electrons. It simplifies the exact many-particle potential by an average or mean potential ${ }^{2}$ that transforms the many-body Schrödinger equation into a much more simple set of time-independent single-particle Schrödinger equations. The Hartree-Fock method ${ }^{4,5}$ is a successful example of such approximation. However, by construction, the correlations among electrons can only be treated approximately. In principle, the density-functional theory ${ }^{6,7}$ provides an exact path to deal with full electron correlations using single-particle potentials. However, since the exact form of the single-particle potentials ${ }^{6-9}$ is unknown, an educated guess for these average single-particle potentials is needed in all practical algorithms. Therefore, again, the electron-electron correlations are treated approximately..$^{9,10}$

The accurate treatment of the electron-electron correlations in electric circuits is even a more difficult issue ${ }^{11-26}$ because we deal with nonequilibrium open systems ${ }^{11-13}$ (where the system interchanges energy and particles with its environment). In fact, the Coulomb interaction among electrons is directly not considered in many quantum transport formalisms ${ }^{11,12}$ under the assumption that the open system behaves as a Fermi liquid. ${ }^{14}$ The well-known Landauer approach $^{15,16}$ is a very successful example of the applicability of this assumption. Nevertheless, the Fermi-liquid paradigm has difficulties dealing with high-frequency, ${ }^{11,17}$ low-dimensionality, ${ }^{1,2}$ or Coulomb blockade regimes. ${ }^{11,18} \mathrm{On}$ the other hand, the nonequilibrium Green's-function formalism (also referred to as the Keldysh formalism) provides an interesting path to solve the Schrödinger equation with the Coulomb interaction introduced perturbatively. ${ }^{19}$ Alternatively, under the assumption that the system behaves like a capacitor, one can use a simple linear relationship between the number of electrons and the electrostatic potential in a particular region to introduce partially Coulomb effects. ${ }^{12,18}$ The mean-field approximation appears again as a solution for electron transport. For example, an average single-particle time-independent potential profile can be computed, selfconsistently, from the set of wave-function solutions of a single-particle time-independent Schrödinger equation. ${ }^{2,12}$ This represents a zero-order approximation (sometimes called the Hartree ${ }^{4}$ approximation) to the complex problem of electron-electron correlations. Additionally, remarkable efforts have been done by Büttiker and co-workers ${ }^{20-22}$ to include Coulomb interaction in their scattering matrix approach by applying different many-body approximations to provide self-consistent electron-transport theories with overall charge neutrality and total current conservation. Finally, extensions of the equilibrium density-functional theory to deal with electron transport, by means of a time-independent formalism, ${ }^{23}$ or with a powerful time-dependent version ${ }^{24-26}$ can also be found in the literature. The exact exchangecorrelation functionals needed in both formalisms are unknown and they have to be approximated. Therefore, in all the descriptions of nonequilibrium quantum systems mentioned here, the electron-electron correlations are approximated to some extent. 
For classical electron devices, the electrostatic interaction among electrons is commonly obtained from an explicit solution of the Poisson (Coulomb) equation. However, again, this does not provide an exact treatment of the classical electron-electron correlations but only an average estimation. ${ }^{27,28}$ It is well known that the solution of a classical many-particle system can always be written as a coupled system of single-particle Newton-type equations. However, a classical mean-field approximation is explicitly assumed in semiclassical transport simulators in order to deal with a unique average electrostatic potential for all electrons. ${ }^{27} \mathrm{~A}$ successful application of the classical mean-field approximation appears, for example, in the semiclassical Boltzmann equation that describes the time evolution of the electron distribution function in a one-electron phase space. ${ }^{27}$ The use of a unique electric field (i.e., a unique average electrostatic potential) in the Boltzmann equation neglects the correct electron-electron correlations because each electron "feels" its own charge. ${ }^{28-32}$ A powerful time-dependent technique to solve the Boltzmann equation is the semiconductor Monte Carlo method applied to electron devices. ${ }^{27}$

In this work, we are interested in revisiting the computation of an ensemble of Coulomb-interacting particles in an open system without any of the approximations mentioned in the previous paragraphs. With this goal, we have developed an exact many-particle Hamiltonian for Coulomb-interacting electrons in an open system in terms of the solutions of the Poisson equation. To our knowledge, the type of development of the many-particle Hamiltonian proposed in this paper has not been previously considered in the literature because, up to now, it was impossible to handle the computational burden associated with a direct solution of a many-particle Hamiltonian. Here, we present a classical and also a quantum solution of the many-particle Hamiltonian, both of which are applicable to realistic three-dimensional (3D) electron devices. The classical solution is obtained by solving a coupled system of Newton-type equations with a different electric field for each particle. The quantum solution of the many-particle Hamiltonian is obtained from the use of quantum trajectories. ${ }^{33}$ The merit of the quantum solution is certainly remarkable because, nowadays, the computational burden associated with the direct (i.e., without any approximation) solution of the many-particle wave function is only accessible for very few $(2,3, \ldots)$ electrons. ${ }^{1,2}$ Our quantum algorithm is able to treat electron dynamics without any (mean-field or perturbative) approximation in the description of the electrostatic interaction among a larger number $(\sim 50)$ of electrons. In this paper, we present the classical and quantum algorithms together because they solve exactly the same many-particle Hamiltonian and both share many technical details (such a 3D Poisson solver to treat spatialdependent permittivity scenarios) in their implementation into realistic 3D electron devices.

After this introduction, the rest of the paper is divided as follows. In Sec. II, we write the many-particle Hamiltonian for an ensemble of electrons in an open system. We discuss the role of the boundary conditions on the borders of the open system to include the Coulomb interaction between particles inside and outside of the open system in Sec. III. In Sec. IV, we discuss the solution of the many-particle Hamil- tonian in classical scenarios. The path for the quantum solution is provided in Sec. V using quantum (Bohm) trajectories. In Sec. VI, we show the numerical results for the classical and quantum solutions of the many-particle Hamiltonian for nanoscale electron devices and we compare the results with time-dependent mean-field approximation. We conclude in Sec. VII. Appendixes A and B discuss the technical details of the image-charge method and mean-field approximation.

\section{MANY-PARTICLE HAMILTONIAN IN OPEN SYSTEM}

In this section, we develop an exact expression for the many-particle Hamiltonian that describes a set of electrons in an open system. Throughout this paper, we will assume that the magnetic field is negligible and that the particle velocity is small enough to assume a nonrelativistic behavior. In addition, in order to provide a discussion valid for either classical or quantum systems, we will assume spinless particles. Let us clarify that the exchange interaction is always present in a system of identical particles (electrons), but it will not be mentioned in this section because it does not affect explicitly the expression of the (first-quantization) many-particle Hamiltonians discussed here. The exchange interaction is introduced into the symmetry (when electron positions are interchanged) of the many-body wave function. We will briefly revisit this issue in $\mathrm{Sec} . \mathrm{V}$ when dealing with the quantum solution.

\section{A. Many-particle Hamiltonian for a closed system}

First, we start our discussion with a set of $M$ particles in a closed system. The many-particle Hamiltonian contains kinetic plus Coulomb energies,

$H\left(\vec{r}_{1}, \ldots, \vec{r}_{M}, \vec{p}_{1}, \ldots, \vec{p}_{M}\right)=\sum_{k=1}^{M}\left\{K\left(\vec{p}_{k}\right)+\frac{1}{2} \sum_{\substack{j=1 \\ j \neq k}}^{M} q_{k} \cdot V\left(\vec{r}_{k}, \vec{r}_{j}\right)\right\}$.

The factor $\frac{1}{2}$ that appears in the second term of the right-hand side is due to the fact that each two-particle interaction is counted twice [i.e., $V\left(\vec{r}_{k}, \vec{r}_{j}\right)$ is identical to $\left.V\left(\vec{r}_{j}, \vec{r}_{k}\right)\right]$. The condition $j \neq k$ takes into account the obvious restriction that a particle cannot interact with itself. The kinetic energy $K\left(\vec{p}_{k}\right)$ that appears in Eq. (1) is defined, for a classical system, as

$$
K\left(\vec{p}_{k}\right)=\frac{1}{2 \cdot m_{k}}\left(\vec{p}_{k}\right)^{2},
$$

while for a quantum system

$$
K\left(\vec{p}_{k}\right)=-\frac{\hbar^{2}}{2 \cdot m_{k}} \nabla_{\vec{r}_{k}}^{2} .
$$

Let us notice that the position and momentum in Hamiltonian (1) can be either classical variables, $\vec{r}_{k}$ and $\vec{p}_{k}$ in Eq. (2a), or quantum (real-space representation) operators, $\vec{r}_{k}$ and $-i \hbar \nabla_{\vec{r}_{k}}$ in Eq. (2b). In particular, it is important to emphasize that when we refer to $\vec{r}_{k}$ as the electron position, we are not referring to a fixed position but a variable vector. On the 


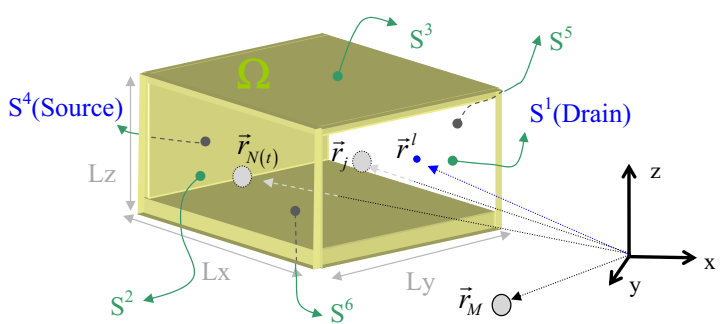

FIG. 1. (Color online) Schematic representation of the volume $\Omega=L x \cdot L y \cdot L z$ and its limiting surface $S=\left\{S^{1}, S^{2}, \ldots, S^{6}\right\}$. There are $N(t)$ particles inside and $M-N(t)$ outside this volume. The vector $\vec{r}$ points an arbitrary position at the boundary surface $S^{l}$.

contrary, when we are interested in specifying a fixed electron position, we will write $\vec{r}_{k}[t]$. The parameter $m_{k}$ is the particle mass that, in Sec. VI, will be understood as the particle effective mass. Identically, we define the Coulomb potential in Eq. (1) as

$$
V\left(\vec{r}_{k}, \vec{r}_{j}\right)=\frac{q_{j}}{4 \pi \varepsilon\left|\vec{r}_{k}-\vec{r}_{j}\right|},
$$

where $q_{j}$ is the particle charge and $\varepsilon$ is the permittivity. Although we are always thinking about electrons in semiconductors, the development of this section is valid for arbitrary particles with different masses and charges.

A complete electronic circuit (including the devices, the wires, and the batteries) behaves as a closed system with a large $(M \rightarrow \infty)$ number of electrons. However, since we can only deal with a finite number of electrons, we restrict our system to a small part of the circuit, for example, the channel of a transistor. Thus, we need to develop the Hamiltonian that describes the dynamics of a subensemble of the whole set of $M$ particles in an open system inside a limited volume $\Omega$ (see Fig. 1).

\section{B. Many-particle Hamiltonian for an open system}

We divide the previous ensemble of $M$ particles into a subensemble $\{1,2,3, \ldots, N(t)\}$ of particles whose positions are inside the volume $\Omega$ and a second subensemble $\{N(t)$ $+1, \ldots, M\}$ which are outside it (see Ref. 34). We assume that the number of particles inside, $N(t)$, is a time-dependent function that provides an explicit time dependence in the many-particle (open-system) Hamiltonian. As drawn in Fig. 1 , we assume a parallelepiped where the six rectangular surfaces $S=\left\{S^{1}, S^{2}, \ldots, S^{6}\right\}$ are the boundaries of $\Omega$. Along this paper, we use $\vec{r}^{l}$ as the "boundary" vector representing an arbitrary position on the surfaces $S^{l}$.

Since we are only interested in the dynamics of the first $N(t)$ particles, the kinetic energy and the Coulomb interaction between the particles of the second subensemble do not appear in the new Hamiltonian of the open system. Nevertheless, the Coulomb interaction between particles of the first and second subensembles must explicitly appear. Thus, the many-particle Hamiltonian for the first $N(t)$ particles can be written as

$$
\begin{aligned}
& H\left(\vec{r}_{1}, \ldots, \vec{r}_{N(t)}, \vec{p}_{1}, \ldots, \vec{p}_{N(t)}, t\right) \\
& \quad=\sum_{k=1}^{N(t)}\left\{K\left(\vec{p}_{k}\right)+\frac{1}{2} \sum_{\substack{j=1 \\
j \neq k}}^{N(t)} q_{k} \cdot V\left(\vec{r}_{k}, \vec{r}_{j}\right)+\sum_{j=N(t)+1}^{M} q_{k} \cdot V\left(\vec{r}_{k}, \vec{r}_{j}\right)\right\} .
\end{aligned}
$$

Let us notice also that the factor $\frac{1}{2}$ disappears in the last term of Eq. (4) because there is no double counting of interactions between electrons inside and outside $\Omega$. For convenience, we rewrite Eq. (4) as

$$
\begin{aligned}
& H\left(\vec{r}_{1}, \ldots, \vec{r}_{N(t)}, \vec{p}_{1}, \ldots, \vec{p}_{N(t)}, t\right) \\
& \quad=\sum_{k=1}^{N(t)}\left\{K\left(\vec{p}_{k}\right)+\sum_{\substack{j=1 \\
j \neq k}}^{N(t)} q_{k} \cdot V\left(\vec{r}_{k}, \vec{r}_{j}\right)+\sum_{j=N(t)+1}^{M} q_{k} \cdot V\left(\vec{r}_{k}, \vec{r}_{j}\right)\right. \\
& \left.\quad-\frac{1}{2} \sum_{j=1}^{N(t)} q_{k} \cdot V\left(\vec{r}_{k}, \vec{r}_{j}\right)\right\} .
\end{aligned}
$$

Although up to this point we have discussed the manyparticle Hamiltonian in terms of the Coulomb force, this approach is inconvenient to deal with solid-state scenarios with a spatial-dependent permittivity. ${ }^{35,36}$ For this reason, we rewrite our many-particle Hamiltonian in terms of the more generic Poisson (or Laplace) equation, which can be applied to systems with (or without) a spatial-dependent permittivity [by substituting $\varepsilon \rightarrow \varepsilon(\vec{r})$ in the Poisson equation].

Each term $V\left(\vec{r}_{k}, \vec{r}_{j}\right)$ that appears in Eq. (5) can be explicitly obtained from a Poisson (or Laplace) equation inside the volume $\Omega$. Then, using the superposition property of the Poisson (or Laplace) equations, we can rewrite Eq. (5) as

$$
\begin{aligned}
& H\left(\vec{r}_{1}, \ldots, \vec{r}_{N(t)}, \vec{p}_{1}, \ldots, \vec{p}_{N(t)}, t\right) \\
& =\sum_{k=1}^{N(t)}\left\{K\left(\vec{p}_{k}\right)+q_{k} \cdot W_{k}\left(\vec{r}_{1}, \ldots, \vec{r}_{N(t)}, t\right)\right. \\
& \left.-\frac{1}{2} \sum_{\substack{j=1 \\
j \neq k}}^{N(t)} q_{k} \cdot V\left(\vec{r}_{k}, \vec{r}_{j}\right)\right\},
\end{aligned}
$$

where the term $W_{k}\left(\vec{r}_{1}, \ldots, \vec{r}_{k}, \ldots, \vec{r}_{N(t)}\right)$ is a particular solution of the following Poisson equation:

$$
\nabla_{\vec{r}_{k}}^{2}\left\{\varepsilon \cdot W_{k}\left(\vec{r}_{1}, \ldots, \vec{r}_{N(t)}\right)\right\}=\rho_{k}\left(\vec{r}_{1}, \ldots, \vec{r}_{N(t)}\right) .
$$

The term $\rho_{k}\left(\vec{r}_{1}, \ldots, \vec{r}_{N(t)}\right)$ in Eq. (7) depends on the position of the first $N(t)$ electrons, ${ }^{34}$

$$
\rho_{k}\left(\vec{r}_{1}, \ldots, \vec{r}_{k}, \ldots, \vec{r}_{N(t)}\right)=\sum_{\substack{j=1 \\ j \neq k}}^{N(t)} q_{j} \cdot \delta\left(\vec{r}_{k}-\vec{r}_{j}\right),
$$

but Eq. (8) is independent of the position of the external particles because they only affect the boundary conditions of Eq. (7). Let us notice that there are still terms, $V\left(\vec{r}_{k}, \vec{r}_{j}\right)$, in 
Eq. (6) that are not computed from Poisson equation but from Eq. (3). However, we will show that these terms $V\left(\vec{r}_{k}, \vec{r}_{j}\right)$ have no role in the classical (i.e., Sec. IV) or quantum (i.e., Sec. V) solutions of Eq. (6).

By construction, comparing Eqs. (5) and (6), the term $W_{k}\left(\vec{r}_{1}, \ldots, \vec{r}_{N(t)}, t\right)$ can be rewritten as

$$
W_{k}\left(\vec{r}_{1}, \ldots, \vec{r}_{N(t)}, t\right)=\sum_{j=1}^{N(t)} V\left(\vec{r}_{k}, \vec{r}_{j}\right)+\sum_{i=N(t)+1}^{M} V\left(\vec{r}_{k}, \vec{r}_{i}\right) .
$$

The dependence of $W_{k}\left(\vec{r}_{1}, \ldots, \vec{r}_{N(t)}, t\right)$ on the positions of the external particles is explicitly written in the last sum in Eq. (9), while in Eq. (7) this dependence is hidden in the boundary conditions of $W_{k}\left(\vec{r}_{1}, \ldots, \vec{r}_{k}, \ldots, \vec{r}_{N(t)}\right)$ on the surface $S$ $=\left\{S^{1}, S^{2}, \ldots, S^{6}\right\}$. In fact, the boundary conditions are a delicate issue that we will discuss in Sec. III. Finally, we want to remark that this discussion is valid for either classical or quantum Hamiltonians because the expression (9) [or its equivalent definition in (7) and (8)] of $W_{k}\left(\vec{r}_{1}, \ldots, \vec{r}_{N(t)}, t\right)$ at $\vec{r}_{1}, \ldots, \vec{r}_{k}, \ldots, \vec{r}_{N(t)}$ is identical for a classical or a quantum system.

\section{BOUNDARY CONDITIONS FOR THE ELECTROSTATIC POTENTIAL $W_{k}\left(\vec{r}_{1}, \ldots, \vec{r}_{k-1}, \vec{r}, \vec{r}_{k+1}, \vec{r}_{N(t)}, t\right)$ ON THE BORDERS OF THE OPEN SYSTEM}

Since we want to deal with solutions of the Poisson equation [Eq. (7)], the boundary conditions for the $N(t)$ terms $W_{k}\left(\vec{r}_{1}, \ldots, \vec{r}_{k}, \ldots, \vec{r}_{N(t)}\right)$ have to be specified on the border surface $S=\left\{S^{1}, S^{2}, \ldots, S^{6}\right\}$. Such boundary conditions will provide, somehow, information on the electrostatic effect that outside electrons [i.e., $N(t)+1, \ldots, M]$ have on the electrons inside $\Omega$. In order to provide a clear notation for discussing the boundary conditions of $W_{k}\left(\vec{r}_{1}, \ldots, \vec{r}_{k}, \ldots, \vec{r}_{N(t)}\right)$, we distinguish between the "source" vectors $\vec{r}_{1}, \ldots, \vec{r}_{k-1}, \vec{r}_{k+1}, \vec{r}_{N(t)}$ and the additional "observation" vector $\vec{r}$ that runs over all space. ${ }^{36}$ In particular, the electrostatic potential that appears in Hamiltonian (6) is defined as the value of the potential $W_{k}\left(\vec{r}_{1}, \ldots, \vec{r}_{k-1}, \vec{r}, \vec{r}_{k+1}, \vec{r}_{N(t)}, t\right)$ at the particular position $\vec{r}=\vec{r}_{k}$,

$$
\begin{aligned}
& W_{k}\left(\vec{r}_{1}, \ldots, \vec{r}_{k-1}, \vec{r}_{k}, \vec{r}_{k+1}, \vec{r}_{N(t)}, t\right) \\
& \quad=\left.W_{k}\left(\vec{r}_{1}, \ldots, \vec{r}_{k-1}, \vec{r}, \vec{r}_{k+1}, \vec{r}_{N(t)}, t\right)\right|_{\vec{r}=\vec{r}_{k}}
\end{aligned}
$$

Our goal is to find an educated guess for all the $N(t)$ terms $W_{k}\left(\vec{r}_{1}, \ldots, \vec{r}_{k-1}, \vec{r}, \vec{r}_{k+1}, \vec{r}_{N(t)}, t\right)$ at all observation points $\vec{r}=\vec{r}$ on all surfaces $l=1, \ldots, 6$. For example, the information of such boundary conditions can come from the value of the total voltage (due to internal and external electrons) at position $\vec{r}^{l}$ and time $t$. We define the total voltage $B\left(\vec{r}_{1}, \ldots, \vec{r}_{N(t)}, \ldots, \vec{r}_{M}, \vec{r}^{l}, t\right)$ as the electrostatic potential associated to an additional probe charge $q_{M+1}$ situated on that boundary, $\vec{r} \equiv \vec{r}_{M+1} \in S^{l}$ (see Fig. 2). The electrostatic potential "seen" by this extra charge due to the presence of the rest of the particles is just

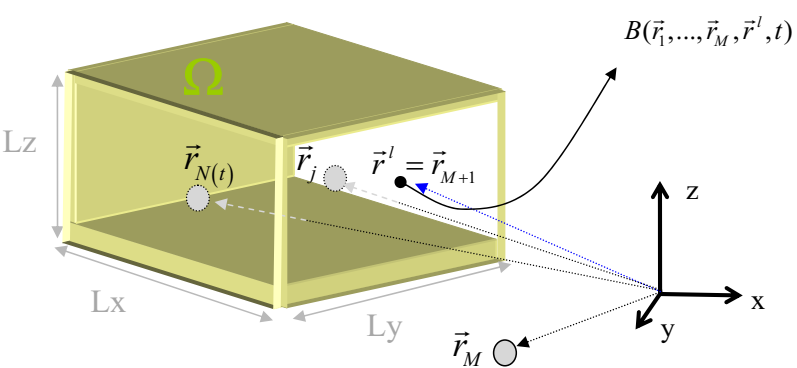

FIG. 2. (Color online) The electrostatic potential $B\left(\vec{r}_{1}, \ldots, \vec{r}_{N(t)}, \ldots, \vec{r}_{M}, \vec{r}^{l}, t\right)$ measured on a surface $S^{l}$ at position $\vec{r}^{l}$ and time $t$ (due to internal and external electrons) by an additional probe charge $q_{M+1}$ situated on the boundary $\vec{r}^{l} \equiv \vec{r}_{M+1} \in S^{l}$.

$$
\left.B\left(\vec{r}_{1}, \ldots, \vec{r}_{N(t)}, \ldots, \vec{r}_{M}, \vec{r}^{l}, t\right) \equiv \sum_{j=1}^{M} V\left(\vec{r}_{M+1}, \vec{r}_{j}\right)\right|_{\vec{r}_{M+1}=\vec{r}^{l},}
$$

where the expected restriction $j \neq M+1$ is hidden in the limit of the sum.

Once relationship (11) is established, we can easily define the boundary conditions of any of the $N(t)$ electrostatic potential $W_{k}\left(\vec{r}_{1}, \ldots, \vec{r}, \ldots, \vec{r}_{N(t)}\right)$ from the function $B\left(\vec{r}_{1}, \ldots, \vec{r}_{N(t)}, \ldots, \vec{r}_{M}, \vec{r}, t\right)$. In particular, from Eq. (9), we know that

$$
\begin{aligned}
& \left.W_{k}\left(\vec{r}_{1}, \ldots, \vec{r}_{k-1}, \vec{r}, \vec{r}_{k+1}, \vec{r}_{N(t)}, t\right)\right|_{\vec{r}=\vec{r}^{l}} \\
& =\sum_{\substack{j=1 \\
j \neq k}}^{M} V\left(\vec{r}^{l}, \vec{r}_{j}\right)=B\left(\vec{r}_{1}, \ldots, \vec{r}_{M}, \vec{r}^{l}, t\right)-V\left(\vec{r}^{l}, \vec{r}_{k}\right), \\
& \quad l=1, \ldots, 6 .
\end{aligned}
$$

The discussion done here is valid for either classical or quantum systems (see Ref. 37). In the previous discussion we have assumed Dirichlet boundary conditions; however it is a straightforwardly procedure to develop the same argumentations for Neumann boundary conditions.

The reader can be surprised by the fact that the right-hand side of expression (12) tends to infinite, $V\left(\vec{r}^{l}, \vec{r}_{k}\right) \rightarrow \infty$, when $\vec{r}_{k} \rightarrow \vec{r}^{l}$. However, when $\vec{r}_{k} \rightarrow \vec{r}^{l}$, the extra particle at $\vec{r}^{l}$ $\equiv \vec{r}_{M+1} \in S_{l}$ will also provide an infinite value of the electrostatic potential, i.e., $B\left(\vec{r}_{1}, \ldots, \vec{r}_{N(t)}, \ldots, \vec{r}_{M}, \vec{r}^{l}, t\right) \rightarrow \infty$, due to the presence of the $k$ particle on the surface. Therefore, the first infinite, $V\left(\vec{r}^{l}, \vec{r}_{k}\right) \rightarrow \infty$, is canceled by the second infinite, $B\left(\vec{r}_{1}, \ldots, \vec{r}_{N(t)}, \ldots, \vec{r}_{M}, \vec{r}, t\right) \rightarrow \infty$. This discussion will be relevant in Sec. VI when we discuss the numerical implementation of these boundary conditions. 


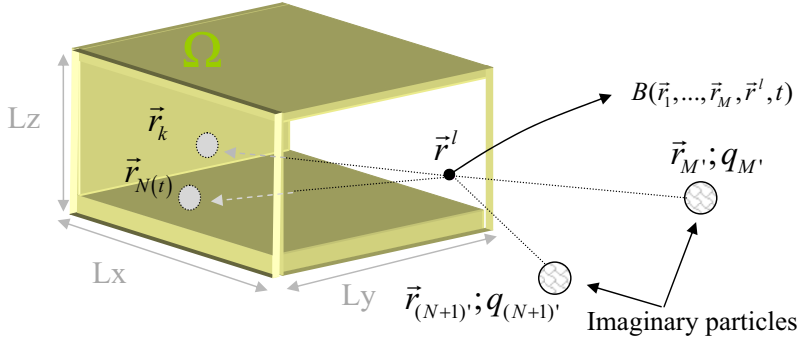

FIG. 3. (Color online) The imaginary charges $q_{(N+1)^{\prime}}$ and $q_{M^{\prime}}$, located outside the volume $\Omega$ at $\vec{r}_{(N+1)^{\prime}}$ and $\vec{r}_{M^{\prime}}$, together with the real particles inside $\Omega$ reproduce the electrostatic potential $B\left(\vec{r}_{1}, \ldots, \vec{r}_{N(t)}, \ldots, \vec{r}_{M}, \vec{r}^{l}, t\right)$ on all $l$ surfaces.

Up to here, our argumentation might seem somehow tricky. We have defined the value of $\left.W_{k}\left(\vec{r}_{1}, \ldots, \vec{r}, \ldots, \vec{r}_{M}\right)\right|_{\vec{r}=\vec{r} l}$ on the volume boundaries without mentioning the position of the external particles but using the function $B\left(\vec{r}_{1}, \ldots, \vec{r}_{N(t)}, \ldots, \vec{r}_{M}, \vec{r}^{l}, t\right)$ which is unknown. This strategy transforms the complexity of finding the boundary conditions of $N(t)$ electrostatic potential $W_{k}\left(\vec{r}_{1}, \ldots, \vec{r}, \ldots, \vec{r}_{N(t)}\right)$ into providing an educated guess for a unique function $B\left(\vec{r}_{1}, \ldots, \vec{r}_{N(t)}, \ldots, \vec{r}_{M}, \vec{r}^{l}, t\right)$. In our numerical results in Sec. VI, we will fix $B\left(\vec{r}_{1}, \ldots, \vec{r}_{N(t)}, \ldots, \vec{r}_{M}, \vec{r}^{l}, t\right)$ based on standard arguments for electron devices. We will assume a uniform value of the voltage on the $l$ surface independent of the external electrons, $B\left(\vec{r}_{1}, \ldots, \vec{r}_{N(t)}, \ldots, \vec{r}_{M}, \vec{r}^{l}, t\right)$ $\approx B^{l}\left(\vec{r}_{1}, \ldots, \vec{r}_{N(t)}, t\right)$. Such value can be obtained taken into account the voltage fixed by the external battery and the requirement of charge neutrality at the contacts..$^{35,38}$

Finally, we want to enlighten the physical interpretation of the many-particle Hamiltonian (6) and boundary conditions of Eq. (12) developed here. To do this, we compare our approach with the image-charge method applied to electron transport. The image-charge method is a basic solving tool in electrostatics ${ }^{36}$ that has been successfully applied, for example, in the calculation of the electric field in field-emission devices ${ }^{39}$ or the barrier-reduction in the metal-semiconductor Schottky contacts. ${ }^{40}$ The name of the method originates from the replacement of certain "real" charges by a set of few "imaginary" charges that replicate the real boundary conditions at the surface (see Fig. 3). From the uniqueness theorem of electrostatics ${ }^{36}$ once the charge of the $1, \ldots, N(t)$ particles inside a volume is fixed and the correct electrostatic potential (or electric field) is specified at the boundaries of that volume, $\left.B\left(\vec{r}_{1}, \ldots, \vec{r}_{N(t)}\right), \ldots, \vec{r}_{M}, \vec{r}^{l}, t\right)$, the solution of the Poisson equation inside the volume is unique and does not depend on whether the external charges are real or imaginary. Then, according to the image-charge method, the electrostatic potential seen by the $k$ particle is equal to the electrostatic potential generated by the sum of the imaginary plus the real particles except the $k$ particle. Thus, identically to our many-particle Hamiltonian, the image-charge method goes beyond the mean-field approximation (discussed in Appendix B) because each particle feels its own potential profile that excludes the Coulomb interaction with itself. In Appendix A, we show in detail that the boundary conditions in
Eq. (12) are identical to the boundary conditions found with the image-charge method.

However, although the outcome of the image-charge method and our many-particle Hamiltonian are identical, the Hamiltonian presented in this paper has an unquestionable advantage over the image-charge method: the former can be directly implemented into practical 3D electron device simulators as we will see in Sec. VI while the latter cannot. For example, let us consider the numerical simulation of the transistor done in Sec. VI. The system consists in a large number ( $\sim 20)$ of electrons inside a volume $\Omega$ limited by surfaces $\left\{S^{1}, S^{2}, \ldots, S^{6}\right\}$ with Dirichlet and Neumann boundary conditions. Then, the exact application of the image-charge method faces up to the following serious difficulties. The computation of the imaginary charges in an arbitrary surface (different from the standard infinite plane whose imaginary charges are found in textbooks ${ }^{41}$ ) is not at all obvious. ${ }^{42}$ Let us notice that each imaginary charge that provides the correct value of the Neumann boundary condition on $S^{i}$ does also affect the Neumann (or Dirichlet) boundary condition on all other surfaces $\left\{S^{1}, S^{2}, \ldots, S^{6}\right\}$. Finally, even after assuming that we would be able to find somehow the density distribution of imaginary charges that reproduces simultaneously the boundary conditions on all six surfaces, the practical application of this method in a time-dependent simulator with a 3D Poisson solver (to be able to deal with spatial-dependent permittivity scenarios) would require simulating much more particles (the real plus the imaginary) in a larger simulation box (to include the location of those imaginary particles outside of $\Omega$ ). In summary, the image-charge method is an excellent approach to obtain analytical expression for the many-body description of electron transport in simple systems (such as one electron crossing an infinite ideal ${ }^{41}$ metallic surface), but it is not practically possible to implement it in simulators for actual $3 \mathrm{D}$ electron devices. ${ }^{42}$ On the contrary, as we will show in our numerical result in Sec. VI, the many-particle Hamiltonian (6) and the boundary conditions of Eq. (12) can be implemented in a extremely simple and transparent way in, either classical or quantum, realistic electron device simulators using a 3D Poisson solver for arbitrary surfaces.

\section{EXACT MANY-PARTICLE HAMILTONIAN FOR CLASSICAL OPEN SYSTEMS}

In this section, we discuss the classical solution of the many-particle open-system Hamiltonian of expression (6). Interestingly, the results obtained here can partially be used for the quantum solution of the many-particle Hamiltonian developed in Sec. V.

The classical description of the particle dynamics subjected to the many-particle Hamiltonian (6) can be computed by using the well-known Hamilton's equations. In particular, we can obtain the (Newton-type) description of the classical trajectory $\vec{r}_{i}[t]$ in the real space through 


$$
\begin{gathered}
\frac{d \vec{p}_{i}[t]}{d t}=\left[-\nabla_{\vec{r}_{i}} H\left(\vec{r}_{1}, \ldots, \vec{r}_{N(t)}, \vec{p}_{1}, \ldots, \vec{p}_{N(t)}, t\right)\right]_{\vec{r}_{1}=\vec{r}_{1}[t], \ldots, \vec{p}_{N(t)}=\vec{p}_{N(t)}[t]}, \\
\frac{d \vec{r}_{i}[t]}{d t}=\left[\nabla_{\vec{p}_{i}} H\left(\vec{r}_{1}, \ldots, \vec{r}_{N(t)}, \vec{p}_{1}, \ldots, \vec{p}_{N(t)}, t\right)\right]_{\vec{r}_{1}=\vec{r}_{1}[t], \ldots, \vec{p}_{N(t)}=\vec{p}_{N(t)}[t]} .
\end{gathered}
$$

For the many-particle Hamiltonian studied in this work, expression (13b) gives the trivial result $m \cdot \vec{v}_{i}[t]=\vec{p}_{i}[t]$, while the evaluation of expression (13a) requires a detailed development. We know that the $\vec{r}_{i}$ gradient of the exact many-particle Hamiltonian (6) can be written as

$$
\begin{aligned}
{\left[\nabla_{\vec{r}_{i}} H\right]_{R}=\vec{R}[t] } & =\left[\nabla _ { \vec { r } _ { i } } \sum _ { k = 1 } ^ { N ( t ) } \left\{q_{k} \cdot W_{k}\left(\vec{r}_{1}, \ldots, \vec{r}_{N(t)}, t\right)\right.\right. \\
& \left.\left.-\frac{1}{2} \sum_{\substack{j=1 \\
j \neq k}}^{N(t)} q_{k} \cdot V\left(\vec{r}_{k}, \vec{r}_{j}\right)\right\}\right]_{\vec{R}=\vec{R}[t]} .
\end{aligned}
$$

We define the multidimensional vector $\vec{R}=\left(\vec{r}_{1}, \ldots, \vec{r}_{N(t)}\right)$ to account, in a compact way, for the classical trajectories of $N(t)$ electrons, $\vec{R}[t]=\left(\vec{r}_{1}[t], \ldots, \vec{r}_{N(t)}[t]\right)$. Substituting the definition of $W_{k}\left(\vec{r}_{1}, \ldots, \vec{r}_{N(t)}, t\right)$ done in expression (9) into Eq. (14), we find

$$
\begin{aligned}
{\left[\nabla_{\vec{r}_{i}} H\right]_{\vec{R}=\vec{R}[t]} } & {\left[\nabla_{\vec{r}_{i}}\left\{2 \sum_{\substack{j=1 \\
j \neq i}}^{N(t)} q_{j} V\left(\vec{r}_{j}, \vec{r}_{i}\right)+\sum_{j=N(t)+1}^{M} q_{j} V\left(\vec{r}_{j}, \vec{r}_{i}\right)\right\}\right.} \\
& \left.-\nabla_{\vec{r}_{i}} \sum_{\substack{j=1 \\
j \neq i}}^{N(t)} q_{j} \cdot V\left(\vec{r}_{j}, \vec{r}_{i}\right)\right]_{\vec{R}=\vec{R}[t]} .
\end{aligned}
$$

Note the elimination of the factor $\frac{1}{2}$ in the last term of the right-hand part of Eq. (15) that accounts for those terms $q_{k} \cdot V\left(\vec{r}_{k}, \vec{r}_{j}\right)$ in Eq. (14), where $\vec{r}_{k} \neq \vec{r}_{i}$ and $\vec{r}_{j}=\vec{r}_{i}$ that are identical to the term $q_{i} \cdot V\left(\vec{r}_{i}, \vec{r}_{k}\right)$ in Eq. (15). For the same reason, we include a factor 2 on the first term of right hand of Eq. (15). From expressions (9) and (15), we realize that

$$
\left[\nabla_{\vec{r}_{i}} H\right]_{\vec{R}=\vec{R}[t]}=\left[\nabla_{\vec{r}_{i}} W_{i}\left(\vec{r}_{1}, \ldots, \vec{r}_{N(t)}\right)\right]_{\vec{R}=\vec{R}[t]} .
$$

Only the term $W_{i}\left(\vec{r}_{1}, \ldots, \vec{r}_{N(t)}\right)$ of whole Hamiltonian (6) becomes relevant for a classical description of the $i$ particle. In fact, since we only evaluate a $\vec{r}_{i}$ gradient, the rest of particle positions can be evaluated at their particular value at time $t$, i.e., $\vec{r}_{k} \rightarrow \vec{r}_{k}[t]$ for all $k \neq i$. Therefore, we define the singleparticle potential $\bar{W}_{i}\left(\vec{r}_{i}, t\right)$ from the many-particle potential as

$$
\bar{W}_{i}\left(\vec{r}_{i}, t\right)=W_{i}\left(\vec{r}_{1}[t], \ldots, \vec{r}_{i-1}[t], \vec{r}_{i}, \vec{r}_{i+1}[t], \ldots, \vec{r}_{N(t)}[t]\right) .
$$

We use a "hat" to differentiate the (time-dependent) singleparticle electrostatic potential from the many-particle poten- tial. Each $i$ term of the single-particle electrostatic potential, $\bar{W}_{i}\left(\vec{r}_{i}, t\right)$, is a solution of one particular 3D-Poisson equation,

$$
\nabla_{\vec{r}_{i}}^{2}\left[\varepsilon\left(\vec{r}_{i}\right) \cdot \bar{W}_{i}\left(\vec{r}_{i}, t\right)\right]=\bar{\rho}_{i}\left(\vec{r}_{i}, t\right),
$$

where the single-particle charge density is defined as

$$
\bar{\rho}_{i}\left(\vec{r}_{i}, t\right)=\sum_{\substack{j=1 \\ j \neq i}}^{N(t)} q_{j} \delta\left(\vec{r}_{i}-\vec{r}_{j}[t]\right),
$$

and the boundary conditions [Eq. (12)] are adapted here as

$$
\begin{gathered}
\left.\bar{W}_{i}\left(\vec{r}_{i}, t\right)\right|_{\vec{r}_{i}=\vec{r}^{l}}=B^{l}\left(\vec{r}_{1}[t], \ldots, \vec{r}_{N(t)}[t], t\right)-V\left(\vec{r}^{l}, \vec{r}_{i}[t]\right), \\
l=1, \ldots, 6 .
\end{gathered}
$$

where we have included the approximation $B\left(\vec{r}_{1}, \ldots, \vec{r}_{N(t)}, \ldots, \vec{r}_{M}, \vec{r}^{\prime}, t\right) \approx B^{l}\left(\vec{r}_{1}, \ldots, \vec{r}_{N(t)}, t\right)$ mentioned in Sec. III. Let us remind that expressions (17)-(20) provide an exact treatment of the many-particle correlations in classical scenarios. They imply a coupled system of Newton equations. The $N(t)$ Newton equations are coupled by $N(t)$ Poisson equations. Therefore, as mentioned in Sec. I, the manyparticle Hamiltonian of Eq. (6) can be written exactly (without mean-field approximation) as a sum of singleparticle Hamiltonian for classical scenarios,

$$
H\left(\vec{r}_{1}, \ldots, \vec{r}_{N(t)}, \vec{p}_{1}, \ldots, \vec{p}_{N(t)}, t\right)=\sum_{k=1}^{N(t)}\left\{K\left(\vec{p}_{k}\right)+q_{k} \cdot \bar{W}_{k}\left(\vec{r}_{k}, t\right)\right\}
$$

The term $\bar{W}_{k}\left(\vec{r}_{k}, t\right)$ in Eq. (21) means that each particle "sees" its own electrostatic potential (or electric field), which is different from that of others.

\section{EXACT MANY-PARTICLE HAMILTONIAN FOR QUANTUM OPEN SYSTEMS}

The many-particle open-system Hamiltonian developed in expression (6) is also valid for quantum systems. In this section, we will explain its practical quantum solution using the recent quantum (Bohm) trajectory formalism in Ref. 33. For convenience, we rewrite the many-particle Hamiltonian in Eq. (6) as 


$$
H\left(\vec{r}_{1}, \ldots, \vec{r}_{N(t)}, t\right)=\left\{\sum_{k=1}^{N(t)}-\frac{\hbar^{2}}{2 \cdot m_{k}} \nabla_{\vec{r}_{k}}^{2}+U\left(\vec{r}_{1}, \ldots, \vec{r}_{N(t)}, t\right)\right\},
$$

where we explicitly write the electron momentum as $\vec{p}_{k}=-i \hbar \nabla_{\vec{r}_{k}}$ in the kinetic energy [as mentioned in Eq. (2b)]. According to Eq. (6), the many-particle electrostatic potential $U\left(\vec{r}_{1}, \ldots, \vec{r}_{N(t)}, t\right)$ is defined as

$$
\begin{aligned}
U\left(\vec{r}_{1}, \ldots, \vec{r}_{N(t)}, t\right)= & \sum_{k=1}^{N(t)}\left\{q_{k} \cdot W_{k}\left(\vec{r}_{1}, \ldots, \vec{r}_{N(t)}, t\right)\right. \\
& \left.-\frac{1}{2} \sum_{\substack{j=1 \\
j \neq k}}^{N(t)} q_{k} \cdot V\left(\vec{r}_{k}, \vec{r}_{j}\right)\right\} .
\end{aligned}
$$

Then, the many-particle time-dependent Schrödinger equation that provides the many-particle wave function, $\Phi\left(\vec{r}_{1}, \ldots, \vec{r}_{N(t)}, t\right)$, which describes the electron dynamics associated to our many-particle (open-system) Hamiltonian, is

$$
\begin{aligned}
i \hbar \frac{\partial \Phi\left(\vec{r}_{1}, \ldots, \vec{r}_{N(t)}, t\right)}{\partial t}= & \left\{\sum_{k=1}^{N(t)}-\frac{\hbar^{2}}{2 \cdot m} \nabla_{\vec{r}_{k}}^{2}\right. \\
& \left.+U\left(\vec{r}_{1}, \ldots, \vec{r}_{N(t)}, t\right)\right\} \cdot \Phi\left(\vec{r}_{1}, \ldots, \vec{r}_{N(t)}, t\right) .
\end{aligned}
$$

The practical utility of expression (24) in understanding quantum scenarios can seem quite doubtful because its direct solution becomes computationally inaccessible for more than very few electrons. ${ }^{1,2,43}$ However, one of the authors has recently developed a transport formalism ${ }^{33}$ in terms of Bohm trajectories that simplifies the complexity of evaluating Eq. (24).

Some introductory explanations about Bohm trajectories in single-particle and many-body scenarios can be found in Refs. 44-47. Here, we go directly to the main result of Ref. 33 where it is shown that a many-particle electron Bohm trajectory $\vec{r}_{a}[t]$ computed from the many-particle wave function, $\Phi\left(\vec{r}_{1}, \ldots, \vec{r}_{N(t)}, t\right)$, solution of the Eq. (24) can be equivalently computed from the single-particle wavefunction $\Psi_{a}\left(\vec{r}_{a}, t\right)$ solution of the following single-particle Schrödinger equation:

$$
\begin{aligned}
i \hbar \frac{\partial \Psi_{a}\left(\vec{r}_{a}, t\right)}{\partial t}= & \left\{-\frac{\hbar^{2}}{2 \cdot m} \nabla_{\vec{r}_{a}}^{2}+U_{a}\left(\vec{r}_{a}, \vec{R}_{a}[t], t\right)+G_{a}\left(\vec{r}_{a}, \vec{R}_{a}[t], t\right)\right. \\
& \left.+i \cdot J_{a}\left(\vec{r}_{a}, \vec{R}_{a}[t], t\right)\right\} \Psi_{a}\left(\vec{r}_{a}, t\right)
\end{aligned}
$$

where we have defined $\vec{R}_{a}[t]=\left\{\vec{r}_{1}[t], \vec{r}_{a-1}[t], \vec{r}_{a+1}[t], \vec{r}_{N}[t], t\right\}$ as a vector that contains all Bohm trajectories except $\vec{r}_{a}[t]$. The exact definition of the other potentials that appear in Eq. (25), $G_{a}\left(\vec{r}_{a}, \vec{R}_{a}[t], t\right)$ and $J_{a}\left(\vec{r}_{a}, \vec{R}_{a}[t], t\right)$, can be obtained from Ref. 33. The total many-particle electrostatic potential in Eq. (24) has been divided into two parts,

$$
U\left(\vec{r}_{a}, \vec{R}_{a}[t], t\right)=U_{a}\left(\vec{r}_{a}, \vec{R}_{a}[t], t\right)+U_{b}\left(\vec{R}_{a}[t], t\right) .
$$

From expressions (9) and (23), we realize that $U_{a}\left(\vec{r}_{a}, \vec{R}_{a}[t], t\right)$ can be greatly simplified as

$$
\begin{aligned}
U_{a}\left(\vec{r}_{a}, \vec{R}_{a}[t], t\right)=2 & \sum_{\substack{j=1 \\
j \neq a}}^{N(t)} q_{a} \cdot V\left(\vec{r}_{a}, \vec{r}_{j}[t]\right)+\sum_{i=N(t)+1}^{M} q_{a} \cdot V\left(\vec{r}_{a}, \vec{r}_{i}[t]\right) \\
& -\sum_{\substack{j=1 \\
j \neq a}}^{N(t)} q_{a} \cdot V\left(\vec{r}_{a}, \vec{r}_{j}[t]\right)=\bar{W}_{a}\left(\vec{r}_{a}, \vec{R}_{a}[t], t\right) .
\end{aligned}
$$

The rest of the terms $V\left(\vec{r}_{j}[t], \vec{r}_{i}[t]\right)$ of expression (26) appear in $U_{b}\left(\vec{R}_{a}[t], t\right)$ and they are included in the potential $G_{a}\left(\vec{r}_{a}, \vec{R}_{a}[t], t\right)$. However, this term $U_{b}\left(\vec{R}_{a}[t], t\right)$ has no role on the single-particle wave function $\Psi_{a}\left(\vec{r}_{a}, t\right)$ because it has no dependence on $\vec{r}_{a}$ and it only introduces an irrelevant global phase on $\Psi_{a}\left(\vec{r}_{a}, t\right)$.

Let us notice that, in the right-hand side of expression (27), we have used the same definition of the potential profile as in classical expression (17). The only difference here is that $\vec{R}_{a}[t]$ are not classical trajectories but quantum (Bohm) trajectories. Therefore, the computation of the potential profile $\bar{W}_{a}\left(\vec{r}_{a}, \vec{R}_{a}[t], t\right)$ that appears in the single-particle Schrödinger equation [Eq. (25)] just needs 3D Poisson equations [Eqs. (18) and (19)] with the boundary conditions [Eq. (20)]. Interestingly, since the term $\bar{W}_{a}\left(\vec{r}_{a}, \vec{R}_{a}[t], t\right)$ is computed from a Poisson equation, our quantum-trajectory algorithm can also be directly extended to spatial-dependent permittivity systems.

In fact, in order to effectively solve the Schrödinger equation [Eq. (25)], we need to know the position of the rest of Bohm particles $\vec{R}_{a}[t]=\left\{\vec{r}_{1}[t], \vec{r}_{a-1}[t], \vec{r}_{a+1}[t], \vec{r}_{N}[t], t\right\}$. Therefore, all $N(t)$ Bohm trajectories have to be computed simultaneously within a system of $N(t)$ Schrödinger equations coupled by $N(t)$ Poisson equations. The keystone of our quantum-trajectory algorithm is that, in order to find $\vec{r}_{a}[t]$, we do not have to evaluate the electrostatic potential, $U\left(\vec{r}_{1}, \ldots, \vec{r}_{N(t)}, t\right)$, and the wave function, $\Phi\left(\vec{r}_{1}, \ldots, \vec{r}_{N(t)}, t\right)$, everywhere in the $N$-multidimensional configuration space $\left\{\vec{r}_{1}, \ldots, \vec{r}_{a}, \ldots, \vec{r}_{N(t)}, t\right\}$, but only over a smaller number of configuration points where all positions of electrons are fixed, $\vec{R}_{a}[t]$, except $\vec{r}_{a}$, i.e., $\left\{\vec{r}_{1}[t], \ldots, \vec{r}_{a}, \ldots, \vec{r}_{N(t)}[t], t\right\}$. We want to remark that the full (short- and long-range) Coulomb interaction present in Eq. (6) is considered explicitly in Eq. (25) without any (mean-field or perturbative) approximation.

Finally, according to the summary done in Sec. I of this work, we want to emphasize the similarities between the (open-system) Bohm-trajectory computational algorithm discussed in Ref. 33 and the (electron-transport version) density-functional theory mentioned in Sec. I. For the latter, the decomposition of the many-particle system into a set of coupled single-particle Schrödinger equations is exact and demonstrated by the Hohenberg-Kohn-Sham theorem. ${ }^{6,7}$ However, from a practical point of view, the exact exchangecorrelation functionals that appear in the single-particle 
Schrödinger equations are unknown and, therefore, require some approximation. Identically, in the quantum (Bohm) trajectory algorithm, the use of single-particle Schrödinger equation [Eq. (25)] is exact to treat many-particle system as demonstrated in the theorem considered in Ref. 33. However, the exact values of the terms $G_{a}\left(\vec{r}_{a}, \vec{R}_{a}[t], t\right)$ and $J_{a}\left(\vec{r}_{a}, \vec{R}_{a}[t], t\right)$ that appear in Hamiltonian (25) are unknown (because they require the partial knowledge of the shape of the many-particle wave function). Thus, from a practical point of view, they need to be approximated by some educated guess as in the density-functional theory.

Finally, the exchange interaction among the (fermions) electrons can also be considered in the present quantum algorithm. A brief explanation of how the exchange interaction can be introduced in the present quantum (Bohm) trajectory algorithm is mentioned in Ref. 33, but the discussion of this issue is far from the goal of the present paper.

\section{NUMERICAL RESULTS FOR THE MANY-PARTICLE HAMILTONIAN IN CLASSICAL AND QUANTUM ELECTRON DEVICES}

In Secs. I-V of this paper, a many-particle Hamiltonian has been developed for an arbitrary ensemble of particles with Coulomb interaction among them. In this section, our numerical examples will deal with electrons in solid-state semiconductors. Therefore, first of all, we have to specify under what approximations the theoretical many-particle Hamiltonian developed in the first part can be used to describe solid-state semiconductors. Only the dynamics of the free electrons will be studied in our numerical results. The interaction with the rest of the charges (associated to core electrons and ions) will be considered as average polarization charges via a spatial-dependent permittivity. ${ }^{36}$ We do also assume an effective mass ${ }^{48}$ for the free electrons that accounts for their interaction with the periodic atomic structure under the standard Born-Oppenheimer approximation ${ }^{49}$ (that neglects the interaction of valence electrons with other kind of particle such as phonons). These are reasonable approximations in most electron-transport models of ballistic devices. ${ }^{12,27}$

We solve the many-particle (open-system) Hamiltonian from expression (6) to compute the current-voltage characteristic for classical and quantum electron devices. We use the classical algorithm discussed in Sec. IV for the simulation of a double-gate field-effect $\operatorname{transistor}^{50}$ (DG-FET), while we use the quantum algorithm discussed in Sec. V for a resonant tunneling diode ${ }^{47}$ (RTD). As mentioned above, no phonon, impurity, or roughness scattering mechanism is included in the simulations and only the full Coulomb interaction is considered explicitly. In order to emphasize the importance of our treatment of the electron-electron correlations in such nanoscale devices, we will compare these current-voltage characteristics with the results obtained with a time-dependent "mean-field" approach that will be discussed in Appendix B. We refer to "many-electron" results to describe the simulation done with either classical (Sec. IV) or quantum (Sec. V) algorithms that requires solving $N(t)$ Poisson equations with $N(t)$ different boundary con- ditions and charge densities [expressions (17)-(20)] at each time step. Alternatively, we refer to the time-dependent "mean-field" results when a single Poisson equation [expressions (B1)-(B4)] is solved for all electrons at each time step of the simulation.

For all simulations (quantum, classical, mean field, or many electron), the same electron injection model is used. We use an injection model applicable to systems with arbitrary electron confinement, which is a time-dependent version of the Landauer boundary conditions, valid for degenerate and nondegenerate systems. We inject electrons according to the Fermi-Dirac statistics defined by a Fermi level (an electrochemical potential) deep inside the contacts. ${ }^{38}$ The applied bias provides a difference between the values of the Fermi level at each injecting surface. Our injection model, coupled to the boundary conditions of the Poisson equation, also ensures charge neutrality at the contacts. $^{35}$

All simulations use a 3D finite-difference Poisson solver scheme. The whole volume $\Omega$ of the active region drawn in Fig. 1 is divided into $N x \cdot N y \cdot N z$ cells. Each $3 \mathrm{D}$ cell has spatial dimensions of $D X, D Y$, and $D Z$. Thus, the active region of our simulating device has a volume equal to $(N x \cdot D X) \cdot(N y \cdot D Y) \cdot(N z \cdot D Z)=L_{x} \cdot L_{y} \cdot L_{z}$. The boundary conditions of the Poisson equation on the six rectangular surfaces $S=\left\{S^{1}, S^{2}, \ldots, S^{6}\right\}$ of Fig. 1 are defined using either Dirichlet or Neumann arguments. In general, on the surfaces $S_{2}, S_{3}, S_{5}$, and $S_{6}$, Neumann boundary conditions are used with the educated guess that the component of the electric field normal to the surface is zero there, $\vec{E}^{l}\left(\vec{r}^{l}, t\right) \cdot \vec{n}^{l}=0$, where $\vec{n}^{l}$ is a unit vector normal to the mentioned surfaces and pointing outward. The continuity of the displacement vector normal to surfaces justifies the assumption $\vec{E}^{l}\left(\vec{r}^{l}, t\right) \cdot \vec{n}^{l}=0$ at the boundaries when the relative permittivity inside $\Omega$ is much higher than the corresponding value outside. On the other hand, in the surfaces $S_{1}$ and $S_{4}$ of Fig. 1 we use the Dirichlet boundary conditions discussed in Sec. III, $B^{l}\left(\vec{r}_{1}, \ldots, \vec{r}_{N(t)}, t\right)$, with final expression (20).

Finally, a technical remark about the application of expression (20) in the classical or quantum many-electron simulations is mandatory. Strictly speaking, our assumption that the potential at one particular surface is position independent, $B\left(\vec{r}_{1}, \ldots, \vec{r}_{N(t)}, \ldots, \vec{r}_{M}, \vec{r}^{l}, t\right) \approx B^{l}\left(\vec{r}_{1}, \ldots, \vec{r}_{N(t)}, t\right)$, is not completely accurate because we known from the discussion in Sec. III that the original function $B\left(\vec{r}_{1}, \ldots, \vec{r}_{N(t)}, \ldots, \vec{r}_{M}, \vec{r}^{l}, t\right)$ in expression (11) has to reproduce, somehow, the atomistic charge distribution on the surface. $^{41}$ In particular, one can expect $B\left(\vec{r}_{1}, \ldots, \vec{r}_{N(t)}, \ldots, \vec{r}_{M}, \vec{r}^{l}, t\right) \rightarrow \infty$ when the electron is close to the border, $\vec{r}_{k} \rightarrow \vec{r}$. However, due to our ignorance about the atomistic description of the contact interface, ${ }^{41}$ we apply the boundary conditions [Eq. (20)] assuming that the distance between $\vec{r}_{k}$ and $\vec{r}^{l}$ is always greater than $1 \mathrm{~nm}$ (this value is interpreted as a measure of range of the atomistic pseudopotential $^{2}$ in the spatial-dependent permittivity scenarios discussed here).

\section{A. Classical simulation of two-electron system: Many electron versus mean field}

In this section we will explain the origin of the important differences that will appear later between the mean-field and 


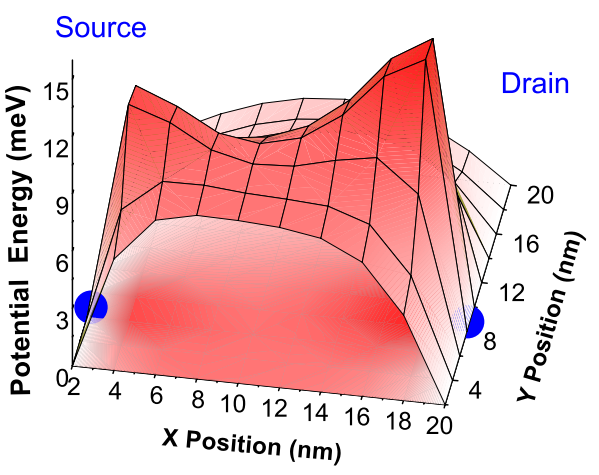

FIG. 4. (Color online) Potential energy profile $\bar{W}_{\text {mean }}(\vec{r}, t)$ computed with a 3D Poisson solver using the classical mean-field approximation on the plane $X-Y$ of the active region $\Omega=(20 \mathrm{~nm})^{3}$ at $z=6 \mathrm{~nm}$ at $0.4 \mathrm{fs}$. The solid points are electron positions.

many-electron algorithms using a simple semiclassical twoelectron system. We consider one electron (labeled as one electron) injected from the source surface, $S_{4}$, at an arbitrary position. A second electron is injected, arbitrarily, from the drain surface, $S_{1}$. A battery provides an external voltage equal to zero at the drain and source surfaces. A 3D cubic system with a volume of $\Omega=(20 \mathrm{~nm})^{3}$ is considered as the active device region. We consider silicon parameters for the numerical simulation. Within the mean-field approximation only the potential profile $\bar{W}_{\text {mean }}(\vec{r}, t)$ is calculated for the twoelectron system using expressions (B1)-(B4). Then, we realize from Fig. 4 that each electron can be reflected by an artificial alteration of the potential profile related to its own charge. In Figs. 5 and 6 we have plotted the energy potential profile seen by the one electron, $\bar{W}_{1}\left(\vec{r}_{1}, t\right)$, and by the two electron, $\bar{W}_{2}\left(\vec{r}_{2}, t\right)$, using the many-electron algorithm described by expressions (17)-(20). Electrons are not affected by their own charge. We clearly see that, within the meanfield approximation, electrons can be unable to overcome the large potential barrier that appears at their own position (due to their own charge). In addition, the simple results confirm that the mean-field error is equal to expression (B7), i.e., the

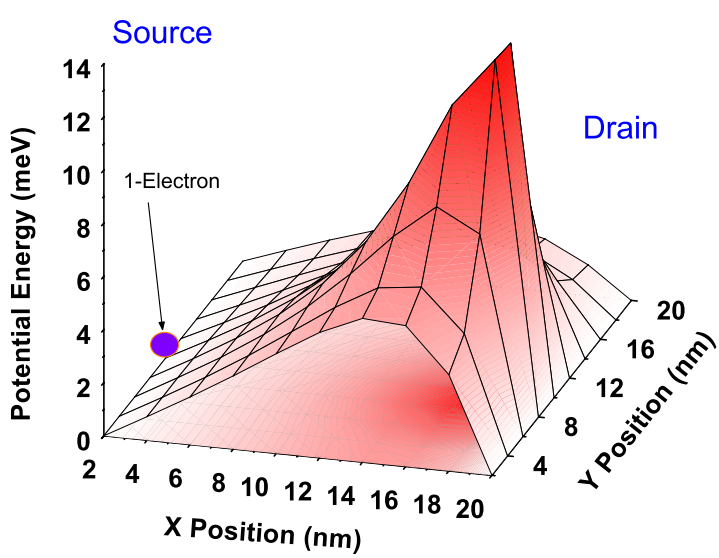

FIG. 5. (Color online) Potential energy profile of the one electron, $\bar{W}_{1}\left(\vec{r}_{1}, t\right)$, with the many-electron algorithm in the plane $X-Y$ of the active region $\Omega=(20 \mathrm{~nm})^{3}$ at $z=6 \mathrm{~nm}$ at $0.4 \mathrm{fs}$. The solid point is the one-electron position.

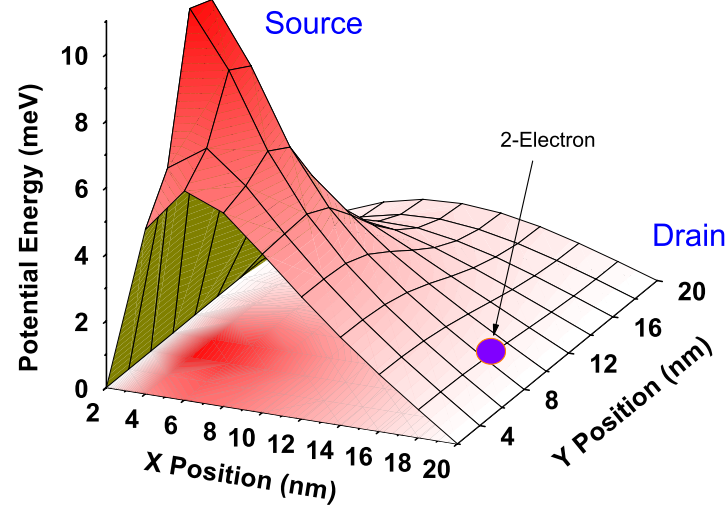

FIG. 6. (Color online) Potential energy profile of the two electron, $\bar{W}_{2}\left(\vec{r}_{2}, t\right)$, with the many-electron algorithm in the plane $X-Y$ of the active region $\Omega=(20 \mathrm{~nm})^{3}$ at $z=6 \mathrm{~nm}$ at $0.4 \mathrm{fs}$. The solid point is the two-electron position.

error of the mean-field potential profile at each position of the active region is $\operatorname{Error}_{k}(\vec{r}, t)=V\left(\vec{r}, \vec{r}_{k}[t]\right)$.

Finally, a discussion about the role of the spatial mesh used for the numerical solution of the Poisson equation is relevant. For an electron device with a length of hundreds of nanometers, we need a mesh of the 3D active region with spatial step $D X \sim D Y \sim D Z>10 \mathrm{~nm}$ to deal with not more than 1000 nodes in the numerical solution of the Poisson equation. This computational limitation in the resolution of the potential is present when solving either the mean-field or the many-electron algorithm. With such spatial resolution, the short-range interaction is missing in both procedures because two electrons inside the same spatial cell will not repel each other. In addition, the error between both procedures, $\operatorname{Error}_{k}(\vec{r}, t)=V\left(\vec{r}, \vec{r}[t]_{k}\right)$, is reduced because the numerical Coulomb potential profile is smoothed due to the low resolution [i.e., the diameter of the region where $V\left(\vec{r}, \vec{r}[t]_{k}\right)$ has a strong influence is shorter than the cell dimensions]. Therefore, we obtain roughly identical results with both schemes. In the subplots of Fig. 7, the same electron trajectory is presented for different mesh resolutions. As can be seen, for the best mesh resolution $(D X=D Y=D Z=2 \mathrm{~nm})$, the differences between both treatments are maximized due to the important spurious autoreflection effect found in the mean-field trajectory. On the other hand, as the resolution of our mesh is reduced, differences between both treatments disappear, giving roughly equal trajectories for cell dimensions above 5 nm.

In summary, when the spatial cells are large, the meanfield and the many-electron schemes correctly model the long-range Coulomb interaction, but both neglect the shortrange component. On the contrary, with smaller spatial steps $D X \sim D Y \sim D Z<5 \mathrm{~nm}$, the many-electron resolution takes into account long- and short-range Coulomb interactions correctly, whereas the description of the short-range component within the mean-field approximation is completely incorrect (i.e., electrons are repelled by themselves). In other words, when $D X, D Y, D Z \rightarrow 0$ the mesh error in our many-electron algorithm reduces to zero, while the error in the mean-field approach tends to infinite, $\operatorname{Error}_{k}(\vec{r}, t) \rightarrow \infty$ (see a schematic summary of the explanation of this discussion in Fig. 8). 


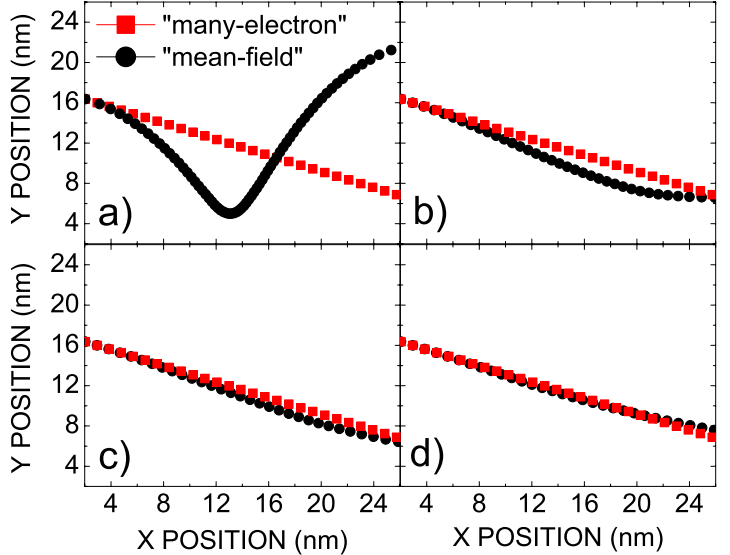

FIG. 7. (Color online) Electron trajectory computed with the mean-field (circles) and the many-electron (squares) algorithms for four different mesh resolutions. (a) $D X=D Y=D Z=2 \mathrm{~nm}$, (b) $D X$ $=D Y=D Z=4 \mathrm{~nm}$, (c) $D X=D Y=D Z=6 \mathrm{~nm}$, and (d) $D X=D Y$ $=D Z=8 \mathrm{~nm}$.

Finally, it is important to remark that the electron trajectories in Fig. 7 are computed using the classical scheme of Sec. IV, but the electrostatic potential profiles are computed from a 3D Poisson solver that is identical for the classical or quantum algorithms. Therefore, the conclusions drawn here for the classical algorithm can be directly extrapolated to our quantum algorithm. In the classical algorithm, the wrong potential profile of Fig. 4 affects the electric field [Eqs. (13a) and (13b)] that modifies the electron dynamics. Identically, the wrong mean-field potential in expression (25) will affect the solution of the Schrödinger equation that will modify Bohm trajectories.

\section{B. Classical simulation of a double-gate field-effect transistor}

Now, we use the classical solution of the many-particle Hamiltonian (6) to provide a full simulation ${ }^{51}$ for the DGFET depicted in Fig. 9. Electron transport in the $x$ direction (from source to drain) takes place along a silicon (100) orientation channel at room temperature. In particular, the electron mass is taken according to the six equivalent ellipsoidal constant energy valleys of the silicon band structure. ${ }^{27,50}$ The effective masses of the ellipsoid are $m_{l}=0.9163 m_{0}$ and $m_{t}$ $=0.1905 m_{0}$, with $m_{0}$ as the free-electron mass. For details on

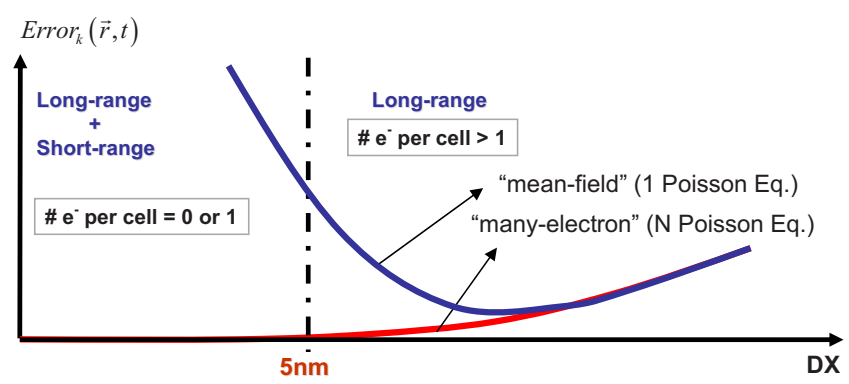

FIG. 8. (Color online) Schematic representation of the errors in the mean-field and the many-electron approaches as a function of the size of the discretization mesh.

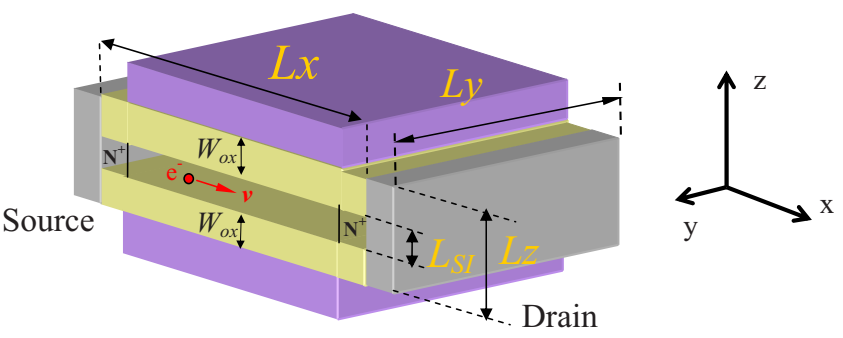

FIG. 9. (Color online) Schematic representation of the DG-FET.

the particular effective mass taken by the electrons in each direction and valley see Ref. 38. The dimensions of the channel of devices $L_{z}$ and $L_{y}$ are both small enough, so that the active region becomes an effective one-dimensional (1D) system (a quantum wire) and the energy of an electron in one particular valley is $E=\hbar^{2} k_{x} /\left(2 m_{t}\right)+E_{1 \mathrm{D}}^{q}$, where $E_{1 \mathrm{D}}^{q}$ $=\hbar^{2} \pi^{2} /\left(2 m_{t} L_{y}^{2}\right)+\hbar^{2} \pi^{2} /\left(2 m_{l} L_{z}^{2}\right)$ represents the minimum energy of the first subband, whose value is $E_{1 \mathrm{D}}^{q}=0.182 \mathrm{eV}$ for $L_{z}=2 \mathrm{~nm}$ and $L_{y}=5 \mathrm{~nm}$. The energies of the next lowest subbands $\left(E_{1 \mathrm{D}}^{q}=0.418 \mathrm{eV}\right.$ or $\left.E_{1 \mathrm{D}}^{q}=0.489 \mathrm{eV}\right)$ are assumed high enough to keep a single band simulation. Therefore, we use a 3D Poisson solver for electrostatic, but a 1D algorithm to describe the velocity of each electron in the $x$ direction. Due to the lateral electron confinement, the velocities in the $y$ and $z$ directions are zero. ${ }^{52}$ We solve $N(t)$ 1D Newton equation [Eq. (13)] coupled by $N(t)$ 3D Poisson equation [Eq. (18)].

We obtain the transistor current-voltage characteristics by computing the time evolution of many interacting electrons inside the 1D DG-FET. The classical many-electron algorithm is compared with the classical mean-field one. The details of the simulation are described in Table I. A total number of cells, $N x \cdot N y \cdot N z$, on the order of 1000 and a number of electrons, $N(t)$, about 20-50, implies a simulation time on the order of $3-4 \mathrm{~h}$ for each bias point, ${ }^{53}$ while it takes 20-30 min within the mean-field approximation.

TABLE I. Parameters for the DG-FET depicted in Fig. 9.

\begin{tabular}{lccc}
\hline \hline & Units & Symbol & Value \\
\hline Lengths & $(\mathrm{nm})$ & $L_{x}$ & 15 \\
& $(\mathrm{~nm})$ & $L_{y}$ & 5 \\
& $(\mathrm{~nm})$ & $L_{z}$ & 6 \\
& $(\mathrm{~nm})$ & $L_{\mathrm{SI}}$ & 2 \\
& $(\mathrm{~nm})$ & $W_{\text {ox }}$ & 2 \\
Spatial step & $(\mathrm{nm})$ & $D X$ & 3.0 \\
& $(\mathrm{~nm})$ & $D Y$ & 1.6 \\
& $(\mathrm{~nm})$ & $D Z$ & 1.0 \\
Relative permittivity & & Air & 1.0005 \\
& & Oxide & 3.8000 \\
& & Silicon & 11.7514 \\
Doping & $\left(\mathrm{cm}^{-3}\right)$ & Channel & Intrinsic \\
& $\left(\mathrm{cm}^{-3}\right)$ & Contact $N^{+}$ & $2 \times 10^{19}$ \\
Simulation time & $\left(\mathrm{sec}^{2}\right)$ & $T$ & $5 \times 10^{-10}$ \\
Temporal step & $\left(\mathrm{sec}^{2}\right)$ & $D t$ & $2 \times 10^{-16}$ \\
\hline \hline
\end{tabular}




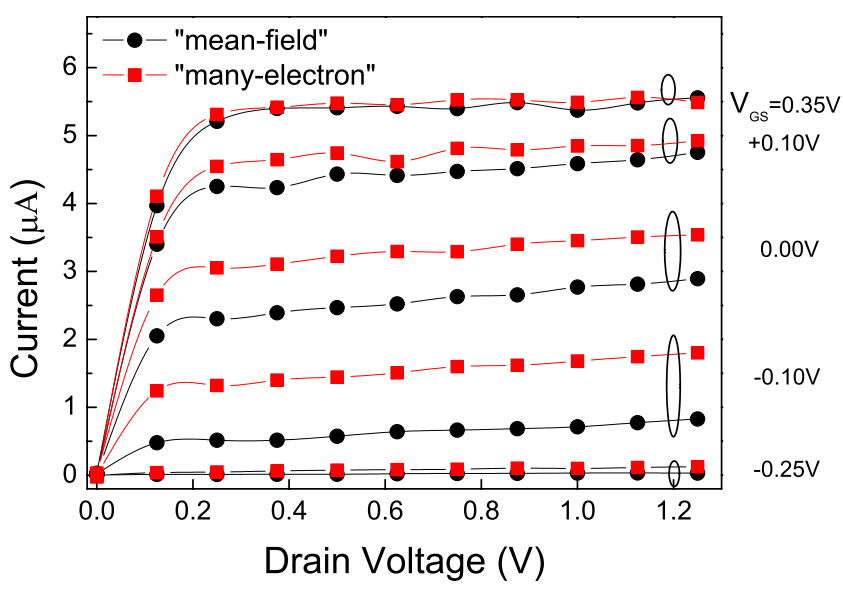

FIG. 10. (Color online) Average current for the 1D DG-FET of Fig. 9 using the many-electron and mean-field algorithms. The open ellipses include results with the same gate voltage indicated on the left.

In Figs. 10 and 11 average values for current and number of particles are presented for different gate and drain voltages. When the FET remains under subthreshold region, the results are quite similar for both methods. However, interestingly, the average current of the FET system in the subthreshold region predicted by the many-electron algorithm is slightly larger than the result obtained by the mean-field approximation. In other words, the mean-field results remain in the subthreshold region, while the many-electron results show a DG-FET channel partially opened. In any case, the most important differences occur for higher gate voltages. In order to understand the results, we have to remind that the DG-FET tends to behave as a capacitor where the charge inside the channel is controlled by the gate voltage. In addition, the charge at the contacts is controlled by the injection process that achieves local charge neutrality there. Therefore, the number of electrons inside the channel tends to be identical within both methods. However, the average current that is sensible to electron dynamics is higher with the many-

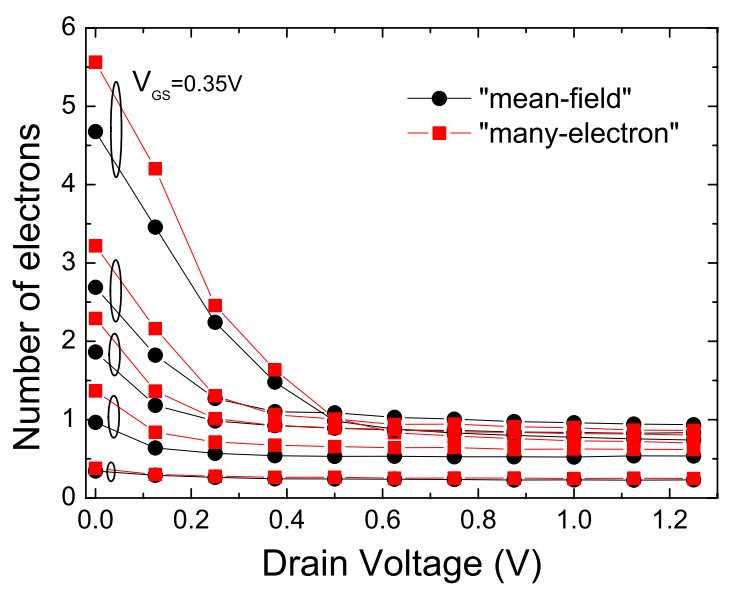

FIG. 11. (Color online) Average number of particles inside the active region of the DG-FET of Fig. 9 using the many-electron and mean-field algorithms. The open ellipses include results with the same gate voltage as indicated on the left of Fig. 10.
TABLE II. Parameters for the RTD depicted in Fig. 12.

\begin{tabular}{lccc}
\hline \hline & Units & Symbol & Value \\
\hline Lengths & $(\mathrm{nm})$ & $L_{x}$ & 6.4 \\
& $(\mathrm{~nm})$ & $L_{y}$ & 9.0 \\
& $(\mathrm{~nm})$ & $L_{z}$ & 9.0 \\
Barrier dimensions & $(\mathrm{eV})$ & High & 0.5 \\
& $(\mathrm{~nm})$ & $L_{\text {well }}$ & 2.4 \\
& $(\mathrm{~nm})$ & $W_{\text {barrier }}$ & 1.6 \\
Relative permittivity & & Air & 1.0005 \\
& & GaAs & 13.1800 \\
& & AlGaAs & 11.7760 \\
Spatial step & $(\mathrm{nm})$ & $D X$ & 0.20 \\
& $(\mathrm{~nm})$ & $D Y$ & 1.12 \\
& $(\mathrm{~nm})$ & $D Z$ & 1.12 \\
Doping & $\left(\mathrm{cm}^{-3}\right)$ & Channel & Intrinsic \\
& $\left(\mathrm{cm}^{-3}\right)$ & Contact $N^{+}$ & $2 \times 10^{19}$ \\
Simulation time & $\left(\mathrm{sec} .^{-}\right)$ & $T$ & $2 \times 10^{-10}$ \\
Temporal step & $(\mathrm{sec})$. & $D t$ & $1 \times 10^{-17}$ \\
\hline \hline
\end{tabular}

electron method than with the mean-field approximation because fewer electrons are reflected in the former (i.e., there are no electrons reflected by its own charge). For the highest gate voltages, equal results for the mean current are obtained for both methods.

\section{Quantum simulation of a resonant tunneling diode}

In this section, we will provide a numerical example of the solution of the quantum many-particle Hamiltonian (6) for an ensemble of electrons in a RTD of Fig. 12. We again compare our many-electron method with the mean-field approximation. We consider a RTD composed of two highly doped drain-source GaAs regions, two AlGaAs barriers, and a quantum well (see Table II). We assume a constant effective mass $m=0.067 m_{0}$, with $m_{0}$ as the electron free mass along the whole structure. Transport takes place from emitter to collector in the $x$ direction. The lateral dimensions are small enough to consider electron confinement in $y$ and $z$ directions. ${ }^{52}$ The energy of an electron in one particular valley is $E=\hbar^{2} k_{x} /(2 m)+E_{1 \mathrm{D}}^{q}$, where $E_{1 \mathrm{D}}^{q}=\hbar^{2} \pi^{2} /\left(2 m L_{y}^{2}\right)$ $+\hbar^{2} \pi^{2} /\left(2 m L_{z}^{2}\right)$ represents the minimum energy of the first subband, whose value is $E_{1 \mathrm{D}}^{q}=0.137 \mathrm{eV}$ for $L_{z}=9 \mathrm{~nm}$ and $L_{y}=9 \mathrm{~nm}$. The energies of the next lowest subbands are inaccessible to electrons $\left(E_{1 \mathrm{D}}^{q}=0.551 \mathrm{eV}\right.$ or $\left.E_{1 \mathrm{D}}^{q}=1.239 \mathrm{eV}\right)$. Again, room temperature is assumed.

The practical quantum algorithm for the RTD implies solving numerically $N(t)$ time-dependent single-particle 1D Schrödinger equation [Eq. (25)] for the transport direction $x$. Due to the confinement in the lateral directions, we assume that the Bohm velocity in $y$ and $z$ directions is negligible. ${ }^{52}$ Since expression (25) deals with time-dependent potential profiles, its solution must be computed with a numerical finite-difference scheme method (see the numerical algorithm presented in Appendix A of Ref. 54). In particular, as discussed in Ref. 33, we assume the zero-order Taylor ap- 


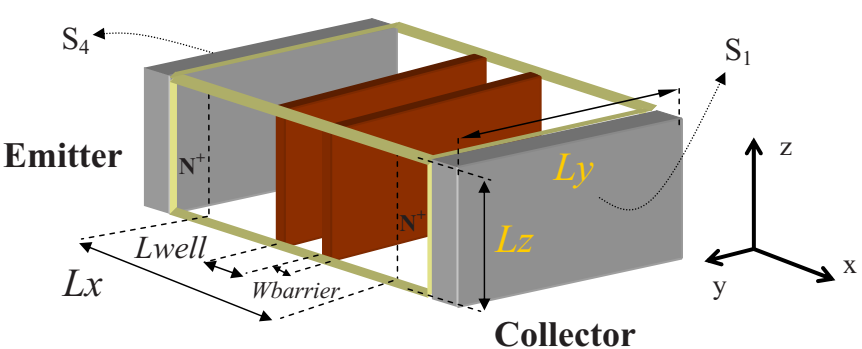
RTD.

FIG. 12. (Color online) Schematic representation of the

proximations $\quad G_{a}\left(\vec{r}_{a}, \vec{R}_{a}[t], t\right) \approx G_{a}\left(\vec{r}_{a}[t], \vec{R}_{a}[t], t\right) \quad$ and $J_{a}\left(\vec{r}_{a}, \vec{R}_{a}[t], t\right) \approx J_{a}\left(\vec{r}_{a}[t], \vec{R}_{a}[t], t\right)$ in expression $(25)$. We do also emphasize that the term $U_{a}\left(\vec{r}_{a}, \vec{R}_{a}[t], t\right)$ that appears in Eq. (25) contains the full (long and short ranges) Coulomb interaction with the particular boundary conditions developed in Sec. III. All Schrödinger equations are coupled by $N(t)$ 3D Poisson equations with $N(t)$ different boundary conditions and charge densities [expressions (17)-(20)]. The total number of cells, $N x \cdot N y \cdot N z$, on the order of 1000 , and the number of electrons, $N(t)$, about 10-20, implies a computational time on the order of 2-3 days for each bias point, ${ }^{53}$ while it takes $10 \mathrm{~h}$ within the mean-field approximation. The calculations of the mean-field approximations and our manyelectron approach are identical except in the computation of the potential profile. In the former a unique potential profile is computed, while in the later there is one potential profile for each electron. Finally, let us notice that we consider resonant tunneling, electron confinement, and Coulomb interaction in our quantum solution of the many-particle Hamiltonian, but we do not include the exchange interaction among electrons. The algorithm to include such interaction in our quantum (Bohm) trajectory proposal is presented in Ref. 33.

In Fig. 13 average values for current are presented for different biases with the many-electron and mean-field algorithms. We compute the average current at each bias point using a detailed version of the Ramo-Shockley theorem ${ }^{54}$ in surface S4 (emitter) and surface S1 (collector). As expected, identical results are obtained from both surfaces showing the numerical accuracy of our simulator. When the RTD remains far from the resonant voltage, the results are quite similar for both methods, but the many-electron approach provides a bit higher current because it avoids the self-reflected electrons in the contact that are found in the mean-field approach, as mentioned previously in Figs. 4-6. On the contrary, in the resonant region, the correct consideration of the electronelectron interaction is very relevant because the quantum transport is very sensible to the quantum well electrostatics. Now, the potential profile determines the shape of the quantum well and, therefore, the resonant energies [dashed horizontal line in insets (a) and (b) of Fig. 13] of the electrons. When a "mean-field" electron tries to traverse the "empty" double barrier structure, it "feels" a perturbation in the quantum well due to its own charge implying an increase in the resonant energy and the possibility of being finally reflected by its own charge. In other words, the "mean-field" electron can be Coulomb blockaded by itself. Our many-electron al-

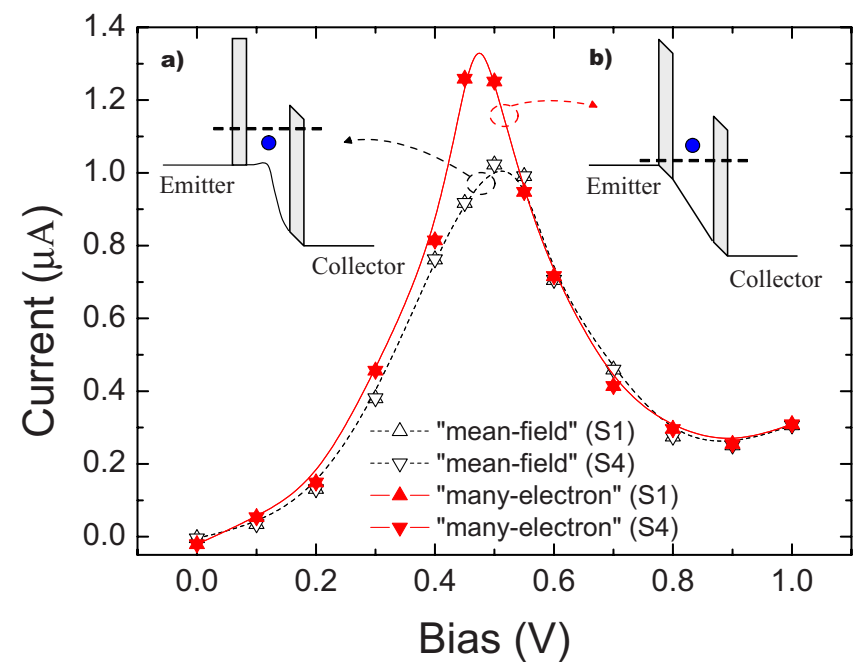

FIG. 13. (Color online) Average current through surfaces S1 and S4 for the RTD of Fig. 12 as a function of bias using the manyelectron (solid symbols) and mean-field (open symbols) algorithms (lines are a visual help). Nonuniform voltages steps are used to focus on the resonant region. Insets show schematically the effect of an electron crossing an empty well on its own electrostatic potential using (a) the mean-field or (b) the many-electron approaches.

gorithm is free from this pathological behavior. This important difference explains the spurious reduction in the current with the mean-field method at resonance. It also explains the movement of the position of the resonant voltage (i.e., the voltage at the maximum current) as schematically explained in the insets of Fig. 13. The inset of Fig. 13(a) shows how the electron that traverses an empty quantum well feels its own repulsion when the mean-field approximation is used (increasing the resonant energy in dotted line), while in inset of Fig. 13(b), the electron with a many-electron simulation is free from this pathological effect.

In summary, the relation between the shape of the potential profile and the behavior of the electron can be much more complex in the quantum regime than in its classical counterpart (where the spatial derivative of the potential profile directly defines the electron acceleration). Figure 13 shows the importance of providing the exact many-particle Coulomb description of electrons in the current-voltage characteristic of a RTD. The time-dependent mean-field approach used here provides spurious effects on the correlations of electrons that are evident even in the dc behavior of RTD simulations. To be fair, let us notice that the time-dependent mean-field approach (the same as in Ref. 47) does improve the treatment of the Coulomb correlations when compared with the standard Fermi-liquid approaches ${ }^{13,14}$ because, in spite of providing a pathological "autointeraction" with itself, it captures the Coulomb correlation between one electron and the others. ${ }^{47}$ In any case, the many-electron approach developed here, with the exact description of the electron Coulomb interactions, is greatly preferred.

\section{CONCLUSIONS}

The prediction of the collective behavior of many electrons is a very active field of research and several theoretical 
approximations have been proposed to improve the treatment of electron-electron correlations. In this work, an exact many-particle Hamiltonian for $N(t)$ electrons inside an open system is developed, without any mean-field or perturbative approximation. The many-particle Hamiltonian (6) is built from a sum of $N(t)$ electrostatic potentials $W_{k}\left(\vec{r}_{1}, \ldots, \vec{r}_{k}, \ldots, \vec{r}_{N(t)}\right)$ solutions of $N(t)$ Poisson equation [Eq. (7)] in a $3 \mathrm{D}$ volume. We use the Poisson equation to define $W_{k}\left(\vec{r}_{1}, \ldots, \vec{r}_{k}, \ldots, \vec{r}_{N(t)}\right)$ instead of the Coulomb law because the former is valid for scenarios with (or without) a spatial-dependent permittivity. In particular, it is shown that the boundary conditions [Eq. (12)] are different for each term $W_{k}\left(\vec{r}_{1}, \ldots, \vec{r}_{k}, \ldots, \vec{r}_{N(t)}\right)$. It is shown that these particledependent boundary conditions [Eq. (12)] of the electrostatic potentials provide the same electron dynamics than the image-charge method applied to electron transport. However, our many-particle approach has the fundamental advantage that it can be directly implemented into 3D realistic (classical or quantum) electron device simulators, while the imagecharge method is an excellent analytical approach applicable only to very simple systems (such as one electron crossing an ideal ${ }^{41}$ infinite metallic surface).

A classical solution of the many-particle Hamiltonian is presented for a DG-FET. The results are compared with a time-dependent mean-field approach described in Appendix B. Within the mean-field approximation only one potential profile $\bar{W}_{\text {mean }}(\vec{r}, t)$ is calculated for all electrons. Then, each electron can be reflected by an artificial alteration of the potential profile due to its own charge. On the contrary, in the many-electron algorithm described here, electrons are not affected by their own charge. The average current and the number of particles are computed for the DG-FET showing that the differences between the mean-field approximation and the exact many-electron approach become important when small geometries (that imply stronger electrostatic interaction) are involved.

A quantum solution of the many-particle Schrödinger equation with the exact many-particle (open-system) Hamiltonian developed here is presented in terms of the quantum (Bohm) trajectory algorithm mentioned in Ref. 33. The relevant point of the quantum-trajectory formalism is that Bohm trajectories can be computed without the full knowledge of the many-particle wave function, $\Phi\left(\vec{r}_{1}, \ldots, \vec{r}_{N(t)}, t\right)$, but with the knowledge of the single-particle wave function, $\Psi_{a}\left(\vec{r}_{a}, t\right)$. It is emphasized that the approach presented in Ref. 33 has similarities with the density-functional theory. In both, the decomposition of many-particle system into a coupled set of single-particle Schrödinger equations is exact, but both need an approximation for the single-particle potentials that appear in their equations [i.e., the exchangecorrelation functionals in the latter and the terms $G_{a}\left(\vec{r}_{a}, \vec{R}_{a}[t], t\right)$ and $J_{a}\left(\vec{r}_{a}, \vec{R}_{a}[t], t\right)$ in the former $]$. We do also emphasize that the electrostatic term $U_{a}\left(\vec{r}_{a}, \vec{R}_{a}[t], t\right)$ that appears in the time-dependent Schrödinger equation with timedependent potentials, expression (25), contains the full (long and short ranges) Coulomb interaction with the particular boundary conditions developed in Sec. III. Numerical results are presented for a RTD and compared with time-dependent mean-field approach developed in Appendix B. The many- electron approach developed here is greatly preferred because it avoids the "self-interaction" found in the timedependent mean-field approach discussed in Appendix B.

Finally, since either the classical or the quantum manyelectron solutions of the Hamiltonian are time-dependent Coulomb-interacting algorithms, apart from the average (dc) current shown in this work, both many-particle approaches are a really valuable simulation tools to obtain reliable information on the high-frequency and ( $\mathrm{dc}$ and ac) noise performances of the state-of-the-art nanoscale devices. Future work will follow this direction.

\section{ACKNOWLEDGMENT}

This work has been partially supported by the Ministerio de Ciencia y Tecnología through Project No. TEC200613731-C02/MIC.

\section{APPENDIX A: THE ELECTRON DYNAMICS OBTAINED BY THE BOUNDARY CONDITIONS [EQ. (12)] AND BY THE IMAGE-CHARGE METHOD}

In this appendix, we show that, in principle, the electrostatic potential that guides the dynamics of the $i$ particle obtained by the image-charge method is identical to the one computed with our many-particle Hamiltonian. In fact, we only have to show that the boundary conditions are identical in both approaches.

As seen in Fig. 3 of the paper, we define a subensemble of imaginary particles $\left\{(N+1)^{\prime}, \ldots, M^{\prime}\right\}$ located outside $\Omega$. The essential property of these imaginary particles is that, together with the first $N(t)$ particles inside $\Omega$, they reproduce the expected value of the potential $B\left(\vec{r}_{1}, \ldots, \vec{r}_{M(t)}, \vec{r}^{l}, t\right)$ measured at the boundary surface $S^{l}$,

$$
\sum_{k=1}^{N(t)} V\left(\vec{r}^{l}, \vec{r}_{k}\right)+\sum_{j^{\prime}=N(t)+1}^{M^{\prime}} \frac{q_{j^{\prime}}}{4 \pi \varepsilon\left|\vec{r}^{l}-\vec{r}_{j^{\prime}}\right|}=B\left(\vec{r}_{1}, \ldots, \vec{r}_{M(t)}, \vec{r}^{l}, t\right),
$$

where the primes $M^{\prime}, j^{\prime}$ in the second term remind that we deal with imaginary charges (the generalization to a distribution of imaginary charge, rather than point particles, is also available). Then, the electrostatic potential seen by the $i$ particle at the $S^{l}$ boundary is just

$$
\left.\bar{W}_{i}^{\text {image }}(\vec{r}, t)\right|_{\vec{r}=\vec{r}^{l}}=\sum_{\substack{k=1 \\ k \neq i}}^{N(t)} V\left(\vec{r}^{l}, \vec{r}_{k}\right)+\sum_{j^{\prime}=N(t)+1}^{M^{\prime}} \frac{q_{j^{\prime}}}{4 \pi \varepsilon\left|\vec{r}^{l}-\vec{r}_{j^{\prime}}\right|} .
$$

Therefore, from Eqs. (A1) and (A2), the electrostatic potential of the $i$ particle at the boundary can be computed as

$$
\left.\bar{W}_{i}^{\text {image }}(\vec{r}, t)\right|_{\vec{r}=\vec{r} l}=B\left(\vec{r}_{1}, \ldots, \vec{r}_{M(t)}, \vec{r}^{l}, t\right)-V\left(\vec{r}^{l}, \vec{r}_{i}\right) .
$$

Expression (A3) is exactly the same result that we obtain from the use of our many-particle boundary conditions [see expression (12)]. Since the charge distribution inside the volume $\Omega$ does not change with the image-charge method or 
with our algorithm and both have identical boundary conditions, the uniqueness electrostatic theorem guarantees that the potential distributions obtained by both algorithms are identical not only in the boundaries but in any point inside the volume $\Omega$. Therefore, we obtain the identity $\bar{W}_{i}^{\text {image }}(\vec{r}, t)$ $=\bar{W}_{i}(\vec{r}, t) ; \forall \vec{r} \in \Omega$. An explanation in terms of the electric field, rather than the electrostatic potential, follows identical steps.

Finally, as mentioned in the paper, let us remind that the demonstration of the identity is quite simple, but the relevant point to compare both methods is that finding the imaginary charges, $\sum_{j^{\prime}=N(t)+1}^{M^{\prime}} q_{j^{\prime}} / 4 \pi \varepsilon\left|\vec{r}^{l}-\vec{r}_{j^{\prime}}\right|$, which fulfill expression (A1) in $3 \mathrm{D}$ realistic scenarios is not at all trivial. ${ }^{41,42}$ On the other hand, the ability of our many-particle Hamiltonian to be included into 3D realistic devices is explicitly demonstrated in Sec. VI.

\section{APPENDIX B: TIME-DEPENDENT MEAN-FIELD APPROXIMATION FOR THE MANY-PARTICLE HAMILTONIAN}

As described in Sec. I, the mean-field approximation provides a single average potential for computing the dynamics of all the electrons. This average potential, which we label here by the suffix "mean" $\bar{W}_{\text {mean }}(\vec{r}, t)$, is still capable of preserving most of the collective effects of the Coulomb interaction. Here, we compare this approximation with our exact many-particle Hamiltonian. The term $\bar{W}_{\text {mean }}(\vec{r}, t)$ is computed by taking into account all charges inside the volume $\Omega$. However, since one particle cannot "feel" its own charge, in fact, $\bar{W}_{\text {mean }}(\vec{r}, t)$ can be interpreted as the electrostatic potential seen for an additional probe charge whose position is $\vec{r}$,

$$
\bar{W}_{\text {mean }}(\vec{r}, t)=\bar{W}_{M+1}\left(\vec{r}_{1}[t], \ldots, \vec{r}_{N(t)}[t], \vec{r}\right) .
$$

This term $\bar{W}_{\text {mean }}(\vec{r}, t)$ is a solution of a unique 3D Poisson equation,

$$
\nabla_{\vec{r}}^{2} \bar{W}_{\text {mean }}(\vec{r}, t)=\bar{\rho}_{\text {mean }}(\vec{r}, t),
$$

where the charge density is defined as

$$
\bar{\rho}_{\text {mean }}(\vec{r}, t)=\sum_{j=1}^{N(t)} q_{j} \delta\left(\vec{r}-\vec{r}_{j}[t]\right),
$$

and, according to expression (11) in the paper, the boundary conditions for this additional probe charge must be

$$
\left.\bar{W}_{\text {mean }}(\vec{r}, t)\right|_{\vec{r}=\vec{r}^{\prime}}=B\left(\vec{r}_{1}[t], \ldots, \vec{r}_{N(t)}[t], \vec{r}^{l}, t\right), \quad l=1, \ldots, 6 .
$$

Let us notice that the time-dependent mean-field approximation discussed here can be applied to either classical or quantum systems. Both approaches share expressions (B2)-(B4) for the computation of the electrostatic potentials (change the classical trajectories by the quantum ones). We also want to remark the time-dependence of expression (B2). This is a common feature for classical (semiconductor Monte
Carlo $^{51}$ ) simulations but less frequent for quantum meanfield approaches.

Now, we estimate the error of our time-dependent meanfield approximation. First, we show that the mean-field potential can be written in terms of the potentials $\bar{W}_{i}\left(\vec{r}_{i}, t\right)$ mentioned in Eq. (17). In particular, we can write the mean-field potential $\bar{W}_{\text {mean }}(\vec{r}, t)$ as

$$
\begin{aligned}
\bar{W}_{\text {mean }}(\vec{r}, t)= & \frac{1}{N(t)}\left\{\sum_{j=1}^{N(t)} \bar{W}_{\text {mean }}(\vec{r}, t)\right\}=\frac{1}{N(t)} \sum_{j=1}^{N(t)}\left\{\bar{W}_{j}(\vec{r}, t)\right. \\
& \left.+V\left(\vec{r}, \vec{r}_{j}[t]\right)\right\} .
\end{aligned}
$$

Now, we compute the error, $\operatorname{Error}_{k}(\vec{r}, t)$, as

$$
\begin{aligned}
\operatorname{Error}_{k}(\vec{r}, t)= & \bar{W}_{\text {mean }}(\vec{r}, t)-W_{k}(\vec{r}, t)=\frac{1}{N(t)}\left\{\sum_{j=1}^{N(t)} \bar{W}_{j}(\vec{r}, t)\right. \\
& \left.+V\left(\vec{r}, \vec{r}_{j}[t]\right)\right\}-\bar{W}_{k}(\vec{r}, t),
\end{aligned}
$$

which can be finally rewritten as

$$
\begin{aligned}
\operatorname{Error}_{k}(\vec{r}, t) & =\frac{1}{N(t)} \sum_{j=1}^{N(t)}\left\{\bar{W}_{j}(\vec{r}, t)-\bar{W}_{k}(\vec{r}, t)+V\left(\vec{r}, \vec{r}_{j}[t]\right)\right\} \\
& =V\left(\vec{r}, \vec{r}_{k}[t]\right),
\end{aligned}
$$

where, according to Eq. (9), we have used the identity

$$
\begin{aligned}
\bar{W}_{j}(\vec{r}, t)-\bar{W}_{k}(\vec{r}, t) & =\sum_{\substack{i=1 \\
i \neq j}}^{M} V\left(\vec{r}, \vec{r}_{i}[t]\right)-\sum_{\substack{i=1 \\
i \neq k}}^{M} V\left(\vec{r}, \vec{r}_{i}[t]\right) \\
& =V\left(\vec{r}, \vec{r}_{k}[t]\right)-V\left(\vec{r}, \vec{r}_{j}[t]\right) .
\end{aligned}
$$

Expression (B7) shows that $\operatorname{Error}_{k}(\vec{r}, t) \rightarrow \infty$ when $\vec{r} \rightarrow \vec{r}_{k}[t]$. The mean-field approximation implies that the potential "felt" by the $k$ particle at $\vec{r} \rightarrow \vec{r}_{k}[t]$ is its own potential profile. In fact, from a numerical point of view, the use of the mean-field approximation is not so bad. For example, classical simulators uses 3D meshes with cell sizes of few nanometers, $D X \approx D Y \approx D Z \gg 10 \mathrm{~nm}$. Then, the error of the meanfield approximation is smaller than the technical error (i.e., mesh error) due to the finite size of the cells. The long-range Coulomb interaction is well captured with the mean-field approximation, while this approximation is really bad strategy to capture the short-range Coulomb interaction ${ }^{28-32}$ (see Fig. 8 in the paper).

Finally, let us remark another important point about the mean-field approximation. Looking at final expression (B7), rewritten here as $W_{k}(\vec{r}, t)=\bar{W}_{\text {mean }}(\vec{r}, t)-V\left(\vec{r}, \vec{r}_{k}[t]\right)$, it seems that $W_{k}(\vec{r}, t)$ can be computed from a unique mean-field solution of the Poisson equation $\bar{W}_{\text {mean }}(\vec{r}, t)$ when subtracting the appropriate two-particle potential $V\left(\vec{r}, \vec{r}_{k}[t]\right)$. In fact, this is the strategy used in several recent works ${ }^{28-32}$ to improve the treatments of the short-range Coulomb interaction in electron device Monte Carlo simulators. However, this strategy is not as general as our procedure because these works 
need an analytical expression for the two-particle Coulomb interaction $V\left(\vec{r}, \vec{r}_{k}[t]\right)$. The analytical expression of $V\left(\vec{r}, \vec{r}_{k}[t]\right)$ written in expression (3) is only valid for scenarios with homogenous permittivity. On the contrary, our procedure with $N(t)$ electrostatic potentials computed from $N(t)$ different Poisson equations in a limited 3D volume $\Omega$ can be applied inside general scenario with (or without) spatialdependent permittivity.
*Corresponding author: xavier.oriols@uab.es

${ }^{1}$ G. F. Giuliani and G. Vignale, Quantum Theory of Electron Liquid (Cambridge University Press, Cambridge, 2005).

${ }^{2}$ J. M. Thijssen, Computational Physics (Cambridge University Press, Cambridge, 2003).

${ }^{3}$ Strictly speaking, even the many-particle wave function of a system of electrons without Coulomb interaction (described by a Slater determinant of single-particle wave functions) is a correlated wave function due to its antisymmetric structure. However, the word correlation is normally reserved only for those correlations that go beyond the inevitable antisymmetry-related one.

${ }^{4}$ D. R. Hartree, Proc. Cambridge Philos. Soc. 24, 89 (1928).

${ }^{5}$ J. C. Slater, Phys. Rev. 81, 385 (1951).

${ }^{6}$ P. Hohenberg and W. Kohn, Phys. Rev. 136, B864 (1964).

${ }^{7}$ W. Kohn and L. J. Sham, Phys. Rev. 140, A1133 (1965).

${ }^{8}$ R. O. Jones and O. Gunnarsson, Rev. Mod. Phys. 61, 689 (1989).

${ }^{9}$ R. O. Jones and O. Gunnarsson, Phys. Rev. Lett. 55, 107 (1985).

${ }^{10}$ D. C. Langreth and M. J. Mehl, Phys. Rev. Lett. 47, 446 (1981).

${ }^{11}$ Y. M. Blanter and M. Büttiker, Phys. Rep. 336, 1 (2000).

${ }^{12} \mathrm{~S}$. Datta, Transport in Mesoscopic Systems (Cambridge University Press, Cambridge, 1995).

${ }^{13}$ W. R. Frensley, Rev. Mod. Phys. 62, 745 (1990).

${ }^{14}$ The absence of Coulomb interaction in electron systems was explained, first, by Landau in terms of the Fermi-liquid paradigm, where the real electrons were substituted by "quasielectrons" with a negligible Coulomb interaction among them: L. D. Landau, Sov. Phys. JETP 8, 70 (1959); L. D. Landau, ibid. 3, 920 (1957).

${ }^{15}$ R. Landauer, IBM J. Res. Dev. 1, 223 (1957).

${ }^{16}$ M. Büttiker, Phys. Rev. Lett. 65, 2901 (1990).

${ }^{17}$ R. Landauer, Phys. Scr., T T42, 110 (1992).

${ }^{18}$ I. L. Aleiner, P. W. Brouwer, and L. I. Glazman, Phys. Rep. 358, 309 (2002).

${ }^{19}$ E. N. Economou, Green's Functions in Quantum Physics (Springer-Verlag, Berlin, 1990).

${ }^{20}$ M. Buttiker, A. Prêtre, and H. Thomas, Phys. Rev. Lett. 70, 4114 (1993).

${ }^{21}$ M. Büttiker, J. Phys.: Condens. Matter 5, 9361 (1993).

${ }^{22}$ M. C. Goorden and M. Büttiker, Phys. Rev. Lett. 99, 146801 (2007).

${ }^{23}$ M. Brandbyge, J. L. Mozos, P. Ordejon, J. Taylor, and K. Stokbro, Phys. Rev. B 65, 165401 (2002).

${ }^{24}$ E. Runge and E. K. U. Gross, Phys. Rev. Lett. 52, 997 (1984).

${ }^{25}$ S. Kurth, G. Stefanucci, C. O. Almbladh, A. Rubio, and E. K. U. Gross, Phys. Rev. B 72, 035308 (2005).

${ }^{26}$ K. Burke, J. Werschnik, and E. K. U. Gross, J. Chem. Phys. 123, 062206 (2005).

${ }^{27}$ C. Jacoboni and L. Reggiani, Rev. Mod. Phys. 55, 645 (1983).

${ }^{28}$ W. J. Gross, D. Vasileska, and D. K. Ferry, IEEE Electron Device Lett. 20, 463 (1999).
${ }^{29}$ S. M. Ramey and D. K. Ferry, Physica B 314, 350 (2002).

${ }^{30}$ S. M. Ramey and D. K. Ferry, IEEE Trans. Nanotechnol. 2, 193 (2003).

${ }^{31}$ P. Dollfus, A. Bournel, S. Galdin, S. Barraud, and P. Hesto, IEEE Trans. Electron Devices 51, 749 (2004).

${ }^{32}$ W. J. Gross, D. Vasileska, and D. K. Ferry, IEEE Trans. Electron Devices 47, 1831 (2000).

${ }^{33}$ X. Oriols, Phys. Rev. Lett. 98, 066803 (2007).

${ }^{34}$ For a quantum system, the number of particles inside the volume $\Omega$ has not only a dependence on time but a dependence on all particle positions $N\left(\vec{r}_{1}, \ldots, \vec{r}_{k}, \ldots, \vec{r}_{M}, t\right)$. Let us assume a wave function whose probability presence occupies regions inside and outside $\Omega$. Then, the limit of the sum in Hamiltonian (6) depends on the exact value of the variable $\vec{r}_{k}$. In principle, the $k$ particle has to be included into the first subensemble when the Hamiltonian deals with $\vec{r}_{k} \in \Omega$ and into the second subensemble when $\vec{r}_{k} \notin \Omega$. In order to avoid a very complicated notation, we do only write the time dependence of $N(t)$ for either classical or quantum systems. In any case, since our quantum solution will deal with quantum (Bohm) trajectories (rather than wave functions), the simpler notation mentioned here is appropriate for the classical and quantum algorithms.

${ }^{35}$ H. López, G. Albareda, X. Cartoixà, J. Suñé, and X. Oriols (unpublished).

${ }^{36}$ M. Javid and P. M. Brown, Field Analysis and Electromagnetics (McGraw-Hill, New York, 1963).

${ }^{37}$ Classically, the definition of $B\left(\vec{r}_{1}, \ldots, \vec{r}_{N(t)}, \ldots, \vec{r}_{M}, \vec{r}^{l}, t\right)$ can be directly associated to the voltage "measured" at $\vec{r}^{l}$ by an external meter. On the contrary, quantum mechanically, the voltage measured at $\vec{r}^{l}$ would require evaluating a bracket between the manyparticle wave function and $B\left(\vec{r}_{1}, \ldots, \vec{r}_{N(t)}, \ldots, \vec{r}_{M}, \vec{r}, t\right)$. In Secs. II and III, we are not discussing the many-particle wave function but only an expression of the many-particle Hamiltonian. Therefore, it is important to remark that $B\left(\vec{r}_{1}, \ldots, \vec{r}_{N(t)}, \ldots, \vec{r}_{M}, \vec{r}^{l}, t\right)$ is not defined as the voltage measured at $\vec{r}^{l}$ but as the electrostatic potential that describes the interaction of the electron at $\vec{r}^{l}$ with the rest of $M$ electrons. This last definition is identical for classical and quantum cases.

${ }^{38}$ X. Oriols, E. Fernandez-Diaz, A. Alvarez, and A. Alarcon, SolidState Electron. 51, 306 (2007).

${ }^{39}$ J. S. Blakemore, Solid State Physics, 2nd ed. (Cambridge University Press, Cambridge, 1985).

${ }^{40}$ S. M. Sze, Semiconductor Devices: Physics and Technology, 2nd ed. (Wiley, New York, 2002).

${ }^{41}$ The standard image-charge method in metallic interfaces assumes that the electric field is null on the interface. However, this assumption fails at very short distances because it does not correctly account for the atomistic structure of the metal and the band structure of its electrons. See section 3.3 of M. W. Finnis, Acta Metall. Mater. 40, S25 (1992); F. Ernst, Mater. Sci. Eng. R 14, 97 (1995). 
${ }^{42}$ Although the image-charge method is an old electrostatics method, its practical application to apparently simple systems has an extraordinary mathematical complexity. See, for example, Z. Jiang, X. Zhang, and J. Han, J. Electrost. 39, 23 (1997), and references therein for its application to two conducting spheres.

${ }^{43}$ W. Kohn, Rev. Mod. Phys. 71, 1253 (1999).

${ }^{44}$ D. Bohm, Phys. Rev. 85, 166 (1952).

${ }^{45}$ P. R. Holland, The Quantum Theory of Motion (Cambridge University Press, Cambridge, 1993).

${ }^{46}$ X. Oriols, Phys. Rev. A 71, 017801 (2005).

${ }^{47}$ X. Oriols, A. Trois, and G. Blouin, Appl. Phys. Lett. 85, 3596 (2004).

${ }^{48}$ J. Wang, A. Rahman, A. Ghosh, G. Klimeck, and M. Lundstrom, IEEE Trans. Electron Devices 52, 1589 (2005).

${ }^{49}$ M. Born and R. Oppenheimer, Ann. Phys. 84, 457 (1927);

${ }^{50}$ V. Sverdlov, X. Oriols, and K. Likharev, IEEE Trans. Nanotechnol. 2, 59 (2003).

${ }^{51}$ Strictly speaking, our many-electron method applied to semiclassical devices cannot be considered as a direct solution of the Boltzmann equation because the latter is developed within a classical mean-field approximation. The term $\bar{W}_{k}\left(\vec{r}_{k}, t\right)$ in the Hamiltonian of expression (21) means that each particle "sees" its own electrostatic potential (or electric field), which is different from that of others. Apart from the scattering rates, this is the fundamental difference between our many-electron method applied to classical transport and the standard Monte Carlo method for electron devices.

${ }^{52} \mathrm{We}$ assume that the electron velocity is equal to zero in the lateral directions where there is energy confinement. This is a reasonable assumption that can be formally justified for Ref. 33 when the probability presence in that direction does not change with time. The main approximation here is assuming that the time dependence of the wave function involves only one quantized energy in the mentioned direction. We define the geometries of the DG-FET and RTD to support these approximations.

${ }^{53}$ The estimation of the computational time is done for simulation run on a single dual xenon $2 \times 3.06 \mathrm{GHz}$ server.

${ }^{54}$ X. Oriols, A. Alarcon, and E. Fernandez-Diaz, Phys. Rev. B 71, 245322 (2005) 